\title{
Experimental Investigation of the Influence of Integral Mechanical Attachments on Structural Behaviour of Timber Folded Surface Structures
}

\author{
Andrea Stitic*, Christopher Robeller, Yves Weinand \\ Laboratory for Timber Constructions, Ecole Polytechnique Federale de Lausanne, \\ CH-1015 Lausanne, Switzerland
}

\begin{abstract}
Structural behaviour of timber folded surface systems greatly depends on the connections ability to transfer the occurring forces between the adjacent elements and finally to the supports. This paper focuses on multiple tab-and-slot joints (MTSJ), where digital prefabrication is used to integrate connectors through plate geometry. Multiple plates assembled within a large scale folded surface structure were tested to examine the influence of connection detail type on its global structural behaviour. For this purpose an innovative test setup was devised that approximates uniformly distributed surface load. The connection details used were chosen with respect to preliminary small scale bending tests. Three groups of distinct large scale structures were tested: 1) structures with miter joint detail and adhesive applied along the edges; 2) structures with open slot MTSJ; and 3) structures with closed slot MTSJ. Extensive investigation into the load bearing behaviour and failure propagation for each of the three different types of structures has been conducted. For analysing their feasibility, the tested structures were also reviewed in terms of fabrication time, assembly and on-site construction. The obtained results show that even though adhesively joined structures provide highest structural stiffness, they exhibit multiple disadvantages when considering building scale applications. Open slot MTSJ structures results indicate that these joints cannot provide sufficiently reliable structural behaviour. Structures with MTSJ closed slots show that their joint geometry greatly improves both the ultimate load-bearing capacity as well as stiffness. Furthermore, by transferring the edge occurring forces mainly in compression, they provide additional ductility to the global system. Within the scope of this paper, closed slot MTSJ proved to be a very efficient connection type which can constitute a robust folded structural system made as a multiple assembly of thin timber plates.
\end{abstract}

Keywords: folded plate, timber panels, multiple tab and slot joint

\section{Introduction}

In structural engineering folded surface structures present one of the concepts for construction of self supporting, column free systems. They utilize structural benefits of folding with regard to material saving and structural efficiency 1]. Additionally, high load-bearing potential and strength to weight ratio of timber panels, all lead to the

\footnotetext{
* Corresponding author

Email address: andrea.stitic@epfl.ch (Andrea Stitic)
}

realization of very efficient lightweight structural systems. As timber folded surface structures consist of a large number of discrete, thin plane elements, proper edgewise connection details are essential for ensuring an efficient load bearing system. For structures made from thin wood panels (thickness/average side ratio: $t / L \leq 0.05$ [2]), such connections present a great challenge. Recently, integral mechanical attachments were proposed by [3, 4, 5, 6] as a new technical solution inspired by traditional woodwork- 
ing joints. Rather than using additional connectors, this technique utilizes digital prefabrication to integrate connectors through the plate geometry. This paper focuses on a particular integral mechanical attachment technique, the so called one-degree-of-freedom multiple tab-and-slot joints (MTSJ). MTSJ geometry can be described with a set of three angles which define the inclination of their locking faces. These angles determine the unique assembly sequence when using such joints in a multiple plate structure. As a result, a geometrical solution for simultaneous joining of adjacent plates with multiple non-parallel edges was presented in [6]. Experimental testing of MTSJ mechanical behaviour suggested that they provide a suitable degree of bending as well as shear stiffness [7, 8]. In these studies the MTSJ semi-rigid behaviour was found to be competitive to that of screwed connections, confirming that they can provide a highly feasible alternative to standard joining techniques. In addition to their good load bearing function, these joints also provide a locator feature for fast and precise positioning of thin elements. The latter being extremely important when multiple, non-parallel plate edges need to be assembled simultaneously. The tests performed by [7, 8] concentrated on individual loading cases, i.e. bending and shear, imposed locally on the MTSJ connection detail. However in the global structure context, where the edge connections are subjected to combined influence of bending, shear, tension and compression, the potential feasibility of such semi-rigid connections has not been studied. It has been demonstrated, regardless of the material, that the connection behavior has a very large influence on the structural performance of civil engineering structures [9, 10]. Therefore, the characterisation of the MTSJ semi-rigidity within a global system is considered to be of crucial importance for establishing timber folded surface structures on a building scale.

This paper examines the influence of the type of connection detail on the global behaviour of folded surface system by performing experimental tests using an innova-

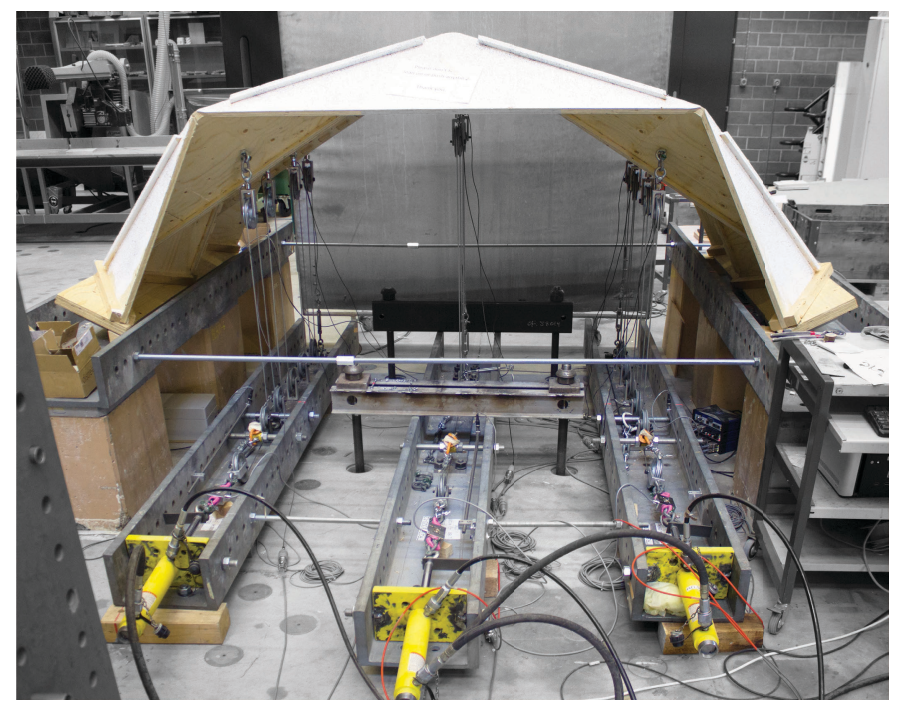

Figure 1: Test setup devised for approximating uniformly distributed surface load.

tive test setup (Fig. 1). It is structured as follows. Section 2 presents the structure design including material, global geometry, connection details and the fabrication process. Section 3 includes preliminary experimental tests on connection details, together with the obtained results and final choice of their parameters, for use in large scale structures. Section 4 presents the test setup and three types of tested large scale structures. Sections 5 and 6 lay out the results and discussions on the large scale tests. Section 7 summarizes the main conclusions. Additionally, appendix $\mathrm{A}$ and $\mathrm{B}$ are included for a more detailed description on the digital fabrication, along with the used test setup and instrumentation.

\section{Structure Design}

Detailed geometry of the test structures was defined considering a series of constraints regarding material, fabrication, connection details and element assembly.

\subsection{Material}

Panel material was chosen as $21 \mathrm{~mm}$ thick Kerto-Q structural grade Laminated Veneer Lumber (LVL). It consists of seven $3 \mathrm{~mm}$ thick spruce peeled-veneer laminates 


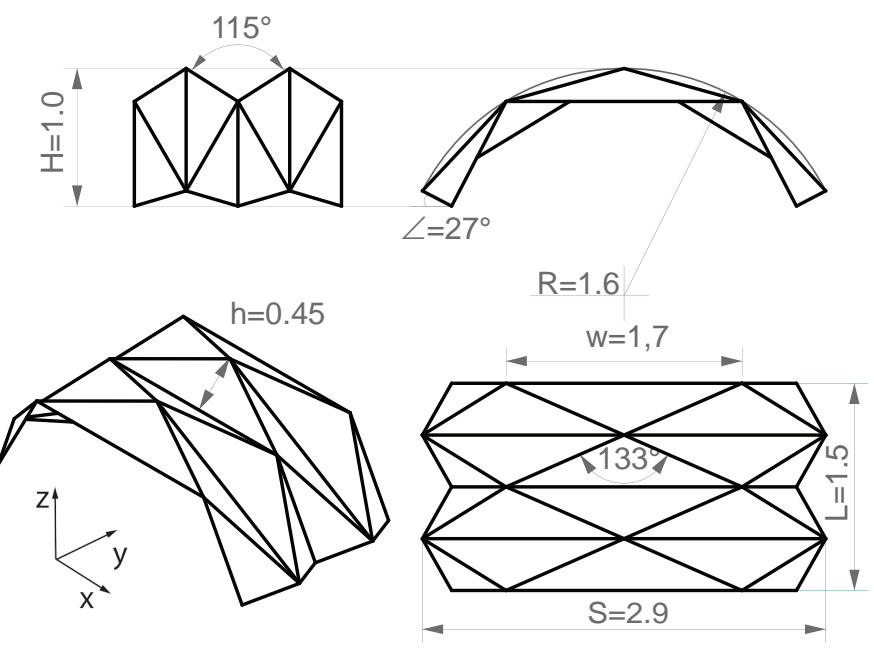

Figure 2: Large scale structure geometry parameters

from which one fifth is glued crosswise in a lay-up | - ||| - |. This kind of composition improves the lateral bending strength and stiffness of the panel. Also, in this way very homogenous and mechanically strong panels are obtained, which can be assumed as having orthotropic material properties [1].

\subsection{Global Geometry}

It has been established that material, fabrication, connection details as well as element assembly constraints, dictate the range of feasible folding angles between adjacent plates, $\varphi$, as well as individual plate geometry [12, 7]. Respectively, the final design of the folded surface was chosen as a regular "Yoshimura" pattern with maximum fold angles equal to $115^{\circ}$ and a transversal cross section following a constant curvature, $R=1,6 \mathrm{~m}$. It consists of twenty discrete elements with maximal plate size of $1,7 m \times 0,45 m$, which form a structure with $3 m$ span in the transversal direction ( $-x$ axis, see Fig. 2 ) and 1,5m length in longitudinal direction $(-y$ axis, see Fig. 2). The height of the structure in its midpoint is equal to $1 \mathrm{~m}$.

\subsection{Connection Details}

In the experimental tests presented in this paper, three different types of structural plate connection details were considered: MTSJ with open slots, MTSJ with closed slots (a)

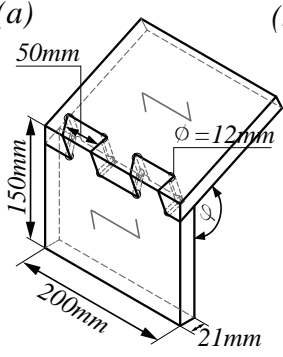

(b)

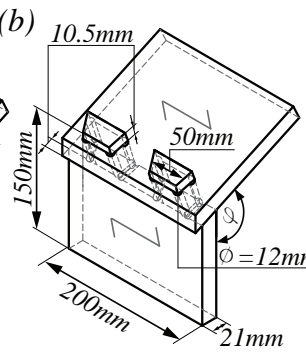

(c)

Figure 3: Test detail geometry parameters, $\varphi=115^{\circ}$; (a) MTSJ with open slots; (b) MTSJ with closed slots; (c) Miter joint.

and adhesively bonded connections (see Fig. 3). The use of metal fasteners was not considered relevant, since their application either highly restricts the requirements for minimal plate thickness, or a large amount of fasteners is necessary for achieving a sufficient connection stiffness [13]. Therefore, in the presented case of edgewise connections between $21 \mathrm{~mm}$ thin plates, such detailing was not feasible.

MTSJ with open slots. These prismatic connections consist of interlocked tabs and slots assembled along a specified vector of insertion. Their geometry can be described by using a set of three Bryant angles, $\theta_{1}, \theta_{2}$ and $\theta_{3}$. They further define the contact locking faces of adjacent edges, as well as the three-dimensional subset of feasible insertion vectors 7]. Their load bearing capacity, i.e. stiffness, greatly depends on the mentioned set of geometrical parameters. Bending and shear load tests, performed on two plate assemblies with various geometries, showed that the highest stiffness of such joints can be expected for the following set of angles: $\theta_{1}=0^{\circ}, 10^{\circ} \leq \theta_{2} \leq 30^{\circ}$, $15^{\circ} \leq \theta_{3} \leq 30^{\circ}[7,8$. These values are further constrained by the requirement for simultaneous assembly of two plate edges where the individual edge insertion vectors have to be parallel [6]. Finally, for such edges, i.e. skewed edges of the triangular plates, angle values were chosen so that they result in insertion vectors parallel to the structure's $-y$ axis; $\theta_{1}=0^{\circ}, \theta_{2}=27^{\circ}, \theta_{3}=20^{\circ}$. Concerning the remaining straight edges, i.e. those parallel to the struc- 


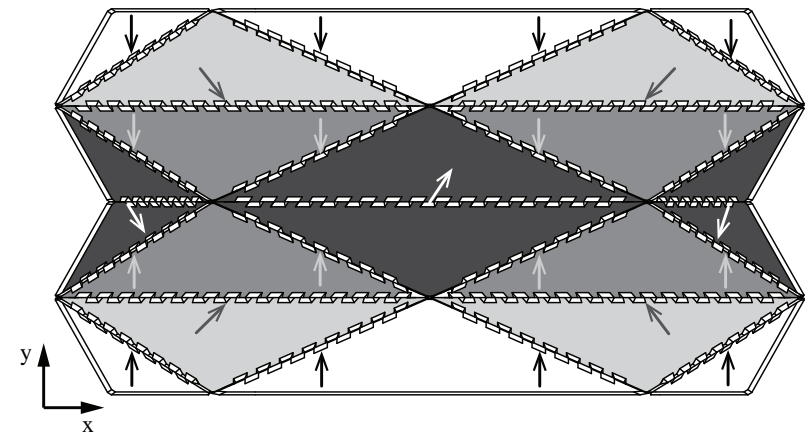

Figure 4: MTSJ plate insertion vectors and assembly sequence of the chosen folded form. Plate grayscale range illustrates the order of assembly, black represents the first and white the last plates to be put in position. Arrows display the insertion vectors.

ture's $-x$ axis, there existed two possibilities for governing the values of their Bryant angles: 1) either the insertion vector of the straight edges is chosen equal to those of the skewed edges, resulting in different values for the straight edges Bryant angles set, $\theta_{1}=0^{\circ}, \theta_{2}=0^{\circ}, \theta_{3}=20^{\circ}$; or 2 ) Bryant angles set values are kept equal to those of skewed edges, resulting in different insertion vector directions for the straight edges. The latter option was chosen in order to maintain equal joint geometry within the entire structure (Fig. 4).

MTSJ with closed slots. In literature, these kind of joints are also referred to as through type joints. Their geometry can be defined in a similar way as for the open slot ones 14, main difference being that their insertion vector is constrained to a two-dimensional subset. This is a result of the slots offset from the plate edge, making all feasible edge insertion vectors lie within the plane of the plate which is being inserted. In the presented case, their assembly sequence is equal to the one of MTSJ with open slots. Additionally, for both MTSJ the length of tabs and slots at the intersection of the plates mid-planes was fixed at $50 \mathrm{~mm}$, to achieve equal distribution along the edges, leaving a maximum of $10 \%$ of the edge length unconnected at the ends.
Adhesively bonded connections. In this detail adhesive was used for realizing edgewise connections which can typically be considered as rigid. This was further used to serve as a reference for determining the level of MTSJ structures semi-rigidity. For achieving the highest possible stiffness various types of edge geometries were tested in combination with 1C PUR glue (Collano Semparoc Rapid-V). The pressure required during curing of the adhesive, for forming the bond between two joining elements, was applied by inserting screws along the edges. The screws were removed before testing and had no influence on the mechanical behaviour of the connections.

\subsection{Digital Fabrication and Assembly}

Manufacturing of individual parts, including the automatic generation of joint geometry with the desired parameters, was done using a digital fabrication tool for generating the 5-axis MAKA MM7S CNC machine G-code. This allowed for the rapid creation of specimens with variable geometry, which would have been impossible with stateof-the-art CAD software tools. These functions were implemented through two custom developed programs, using the programming language Visual $\mathrm{C \#}$ and the Rhino Common Software Development Kit (SDK) 15. A realtime preview of the output geometry was realized through the implementation as a CAD Addon for the visual programming software Grasshopper. For detail description of the used custom tools the reader is referred to appendix A.

Panels of $2,5 m \times 1,25 m$ dimensions were supplied by Metsä-wood Germany. They were cut with a $12 \mathrm{~mm}$ diameter shank-type milling cutter and $0,05 \mathrm{~mm}$ tolerance, creating a tight fit with $0,1 \mathrm{~mm}$ assembly clearance between the adjoining plates. In order to have a smooth assembly, it was necessary to ensure constant $21 \mathrm{~mm}$ thickness of the plates along the edges. As the thickness within one panel may vary up to $\pm 1 \mathrm{~mm}$ [1], each one was planarized along the plate edge joining area before the cut- 
ting process. With respect to the defined insertion vectors and the interlocking advantage of the chosen single-degreeof-freedom MTSJ, it was necessary to follow a specified sequence for assembling individual parts into the global structure (Fig. 4).

\section{Preliminary Connection Detail Tests}

Since bending around the edges is recognised as one of the main and most unfavourable load in timber folded surface structures, small scale bending tests were performed on two plate assemblies in order to establish the detailed parameters of connections to be used in large scale structures. Dimensions of the assembled plates were $200 \mathrm{~mm} \times$ $150 \mathrm{~mm}$ (Fig. 3). The length of the tabs and slots at the intersection of the plates mid-planes was fixed at $50 \mathrm{~mm}$. The plates were positioned under the angle of $\varphi=115^{\circ}$ in a test setup consisting of a fixed part restricting the movement of one plate and a lever arm pushing the other plate. This causes rotation around the central axis of the joined edge and thus closing of the two-plate sample. The details were tested only in the closing mode as it has shown to be less stiff compared to the opening one [7]. Three types of adhesively bonded edge geometries were tested for determining the most rigid one: 1) miter joint with the cut face lying in the internal bisector plane of the joint angle; 2) regular finger joint; and 3) MTSJ with open slots with parameters as explained in Section 2.3 (Table 1). 1C PUR adhesive (Collano Semparoc rapid V) was applied along the edges of adjacent plates and constant pressure during curing was ensured by adding crosswise screws. After 24 hours the screws were removed and the samples tested.

In the interest of examining the failure modes, as well as the level of their semi-rigidity with respect to the glued rigid details, three details without applying adhesive were also tested: 1) MTSJ with open slots with parameters as explained in Section 2.3 2) MTSJ with closed slots and the same parameters; and 3) MTSJ with closed slots where the influence of $\theta_{3}$ angle was studied (Table 1 ).

\subsection{Connections With Adhesive}

Fig. 5 shows the corresponding sample moment-rotation 227 curves obtained from the adhesively bonded test details. The different slopes of the ascending parts of the curves show that the miter joint exhibited the most rigid behaviour. This is contrary to the initial assumption that the combination of adhesive with finger joint or MTSJ geometry would bring benefits with regard to enlarged glued surface area, and therefore stiffness. It is concluded that these benefits are lost due to fabrication constraints as well as necessary tolerances. Such as: the tab and slot sides of two bonded plates cannot be machined precisely enough to achieve the perfect fit needed to distribute pressure uniformly over the entire joint area; moreover, milling sharp corners with a circular tool results in circular notches at the ends of each tab and slot which additionally reduce the adherent's surface length, $l_{a}$ (see Fig. 6).

As shown in (Fig. 6) the miter joints exhibited highly brittle cleavage failure, which took place at the bonded interface. It first occurs in the plate, due to tension perpendicular to the grain in the five layers with grain orientation parallel to the joined edge. The observed failure was very shallow, where only a few wood fibers remained attached to the glue, and it was instantly followed by the adhesive failure in the remaining two layers with opposite grain orientation. On the other hand, failure of the remaining two glued joints happened entirely within the panel, naturally resulting in lower stiffness. In these details the bonding interface is situated between the edges and faces of mutually connected plates, making the bond strength higher. The failure happens due to delamination caused by tension acting perpendicular to the plate plane.

Compared to the glued finger joint, MTSJ exhibited lower stiffness but also a certain level of ductility after failure. This is a result of interlocking due to the introduced $\theta$ angles, where additional compression forces appear between the inclined tab and slot edges. On one hand, this reduces the effective tab length, $l_{e f f}$, for resisting delam- 


\section{Connections with adhesive:}

Miter joint
Finger joint

\section{Connections without adhesive:}

MTSJ open slots MTSJ closed slots MTSJ closed slots

$0_{3}$

Table 1: Connection detail test geometries; three bold lines on adhesively joined details mark the positions of screws used for applying pressure.

ination in the top layers (see Fig 6 ), on the other hand it is responsible for the exhibited post-failure load capacity. Based on the results presented above, the detail with the highest stiffness, i.e. adhesively bonded miter joint, was chosen for application in larger scale structures.

\subsection{Connections Without Adhesive}

Moment-rotation curves of non-glued connection details are also shown in (Fig. 5). They can be generally divided into three parts: 1) first part of the curve shows the relative slip at the joint interface; 2) after coming into full contact the ascending part of the curve describes the joint stiffness; and 3) the descending part describes postfailure behaviour.

With respect to MTSJ with open slots, the ones with closed slots showed higher stiffness and a considerably lower amount of inital slip. This slip is a result of fabrication and assembly tolerances and is significantly reduced for closed slots due to their protrusion geometry. The main failure modes of MTSJ details without adhesives are shown in (Fig. 7). For more details on the mechanical behaviour of the MTSJ with open and closed slots the reader is referred to [16, 7, 14.

Concerning the influence of $\theta_{3}$ angle in MTSJ with closed slots, the results suggest that it has no considerable impact on the stiffness as well as on the strength of the detail. This could be a result of the small plate thickness in respect to relatively large sized notches, where the intended beneficial compression surface at the inclined sides of the joints with $\theta_{3} \neq 0^{\circ}$ was simply too small to make any significant difference in its load-bearing capacity. Consequently, for reasons of simplicity in large scale structures the MTSJ with closed slots was taken with $\theta_{3}=0^{\circ}$.

\section{Large Scale Structure Tests}

Three groups of distinct large scale structures consisting of three replicates were tested, each group with a different connection detail. The details were chosen with respect to the obtained small scale results; 1) miter joint with adhesive applied along the adjoining edges representing a rigid connection; 2) MTSJ with open slots with $\theta_{1}=0^{\circ}$, $\theta_{2}=27^{\circ}, \theta_{3}=20^{\circ}$; and 3) MTSJ with closed slots with $\theta_{1}=0^{\circ}, \theta_{2}=27^{\circ}, \theta_{3}=0^{\circ}$. Additionally, certain adjustments were made concerning the miter joint edge geometry, having seen that already in small scale samples aligning plate edges and inserting screws presented difficulties. One-faced finger joints were added along the edges for ensuring precise positioning during assembly of plates (Fig. 8). They provided space for inserting screws perpendicular to the plate normal direction as well as avoiding sliding while doing so. The specific geometry of these joints allowed for the joint cut face to remain at the internal bisector plane of the dihedral angle, making it possible to integrate with the miter joint edge geometry.

\subsection{Test Setup}

As all surface-active structural systems, folded structures are designed for taking surface loads. Due to the fact 
(a)

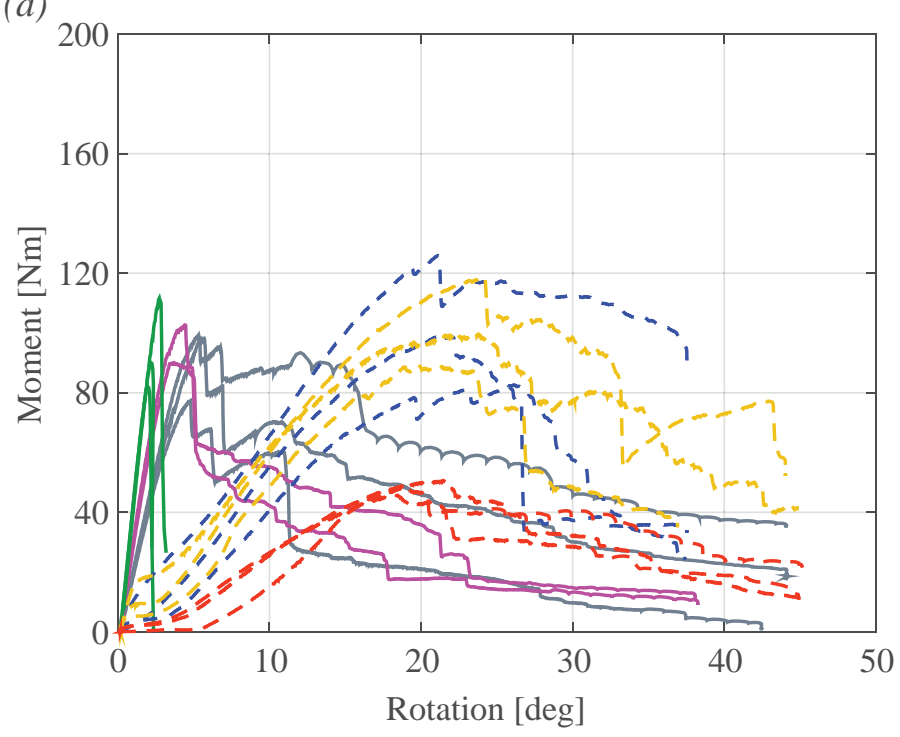

(b)

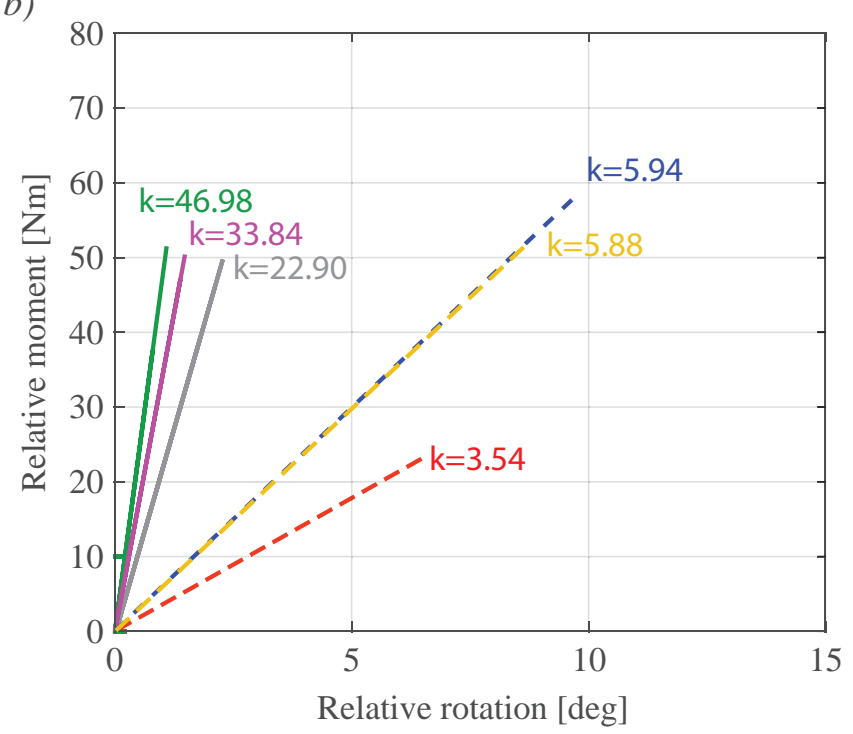

Connection details with adhesive:

— Miter joint Finger joint

\section{MTSJ open slot} $\left(0^{\circ}, 27^{\circ}, 20^{\circ}\right)$

Connection details without adhesive:

- - MTSJ open slot $\left(0^{\circ}, 27^{\circ}, 20^{\circ}\right)$
- MTSJ closed slot $-=-$ MTSJ closed slot $\left(0^{\circ}, 27^{\circ}, 20^{\circ}\right)$ $\left(0^{\circ}, 27^{\circ}, 0^{\circ}\right)$

Figure 5: (a) Moment-rotation curves of 6 connection details. A group of three experimental replicates was produced for each connection detail type and each replicate was fitted with a single curve. Triplets of curves of the same group are marked with the same color; (b) Stiffness, $k$, for each respective group. The coefficient $k$ was determined by fitting a linear regression model to each of the 6 groups containing 3 replicates, in the elastic region of the M-R curve $\left[0.1 M_{\max }, 0.6 M_{\max }\right]$. The lower bound of the elastic region, $0.1 M_{\max }$, was chosen in order to exclude the initial slip while the upper bound, $0.6 M_{\max }$, was determined by imposing $R^{2}>0.95$, where $R^{2}$ is the coefficient of determination of the linear regression.
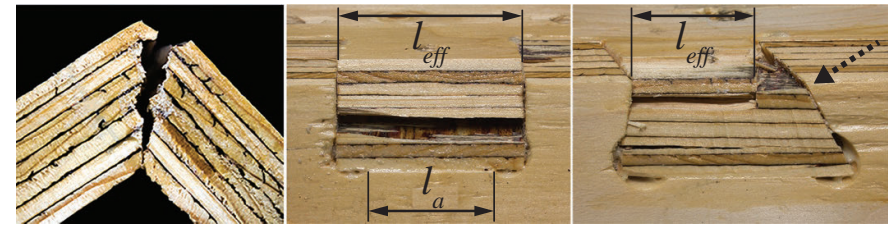

Figure 6: Failure of adhesively bonded connections; from left to right: miter joint, finger joint, MTSJ with open slots. In MTSJ with open slots dashed arrow points to the surface where additional compression occurs. that their surface is corrugated, the application of continuous load in a controlled manner for performing experimental work, has proven to be a challenging task. Similar structures have been tested by applying line and point loads on the structure edges and vertices, where the results showed that in this way a distorted impression of the structural behaviour is obtained, as opposed to that expected in actual practice. It was also assumed that the structures would behave considerably different under a uniformly distributed load [17, 18]. For that, inspiration for alternative experimental methods was found in shell structures. Such methods include the use of pressure as well as vacuum loading techniques or the application of a discrete load systems for simulating uniformly distributed load [19, 20]. Due to the size of our structure as well as its corrugated surface, the use of first two mentioned methods was excluded, due to accuracy issues in constructing an efficient testing apparatus. Finally, it was decided to represent the uniform load with discrete concentrated loads applied at the geometrical center of each plate. For reference, $1 \mathrm{kN}$ of load applied at each of the 10 concentrated points, amounting to $10 \mathrm{kN}$ of total load, was equivalent to $2.63 \mathrm{kN} / \mathrm{m}^{2}$ uniform load. For simplicity, the structure was designed following a singly curved surface in order for all the constituting elements to be of the same shape and size, i.e. 

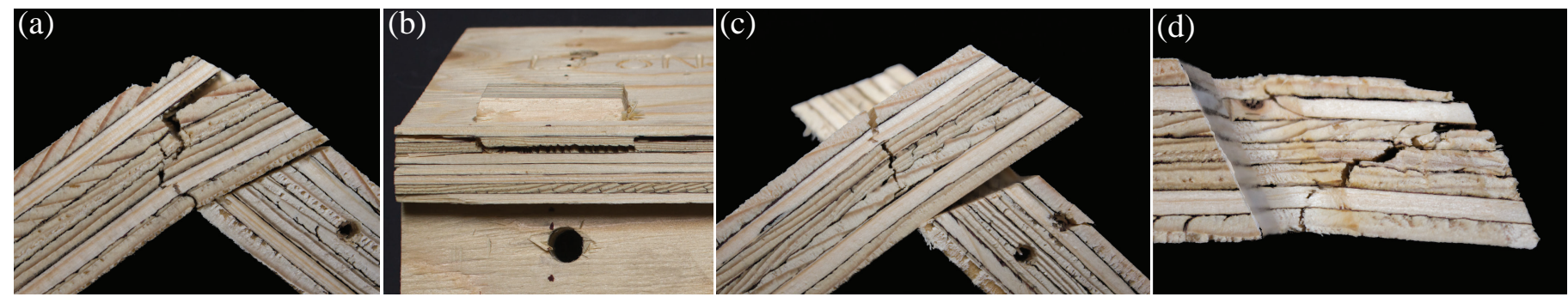

Figure 7: Failure of MTSJ connections without adhesive; (a) MTSJ with open slots showing the side view of tab failure. In two top layers contact is not achieved due to initial slip causing delamination at the interface between 2nd and 3rd layer. The rest of the cross section fails due to tension perpendicular to main plate grain orientation; (b) MTSJ with closed slots, $\theta_{3}=0^{\circ}$. Delamination failure occurs at the interface between differently oriented layers, and failure due to tension perpendicular to grain in layers which are in the direction of plate main grain orientation, i.e. layers $1,3,4,5$ and 7 ; (c) MTSJ with closed slots, $\theta_{3} \neq 0^{\circ}$. Failure at the weakest longitudinal cross section of the slot plate, propagating from the top face due to tension perpendicular to the main plate grain orientation; (d) MTSJ with closed slots, $\theta_{3} \neq 0^{\circ}$. Second type of failure mode, where the tabs fail due to to tension perpendicular to the main plate grain orientation.

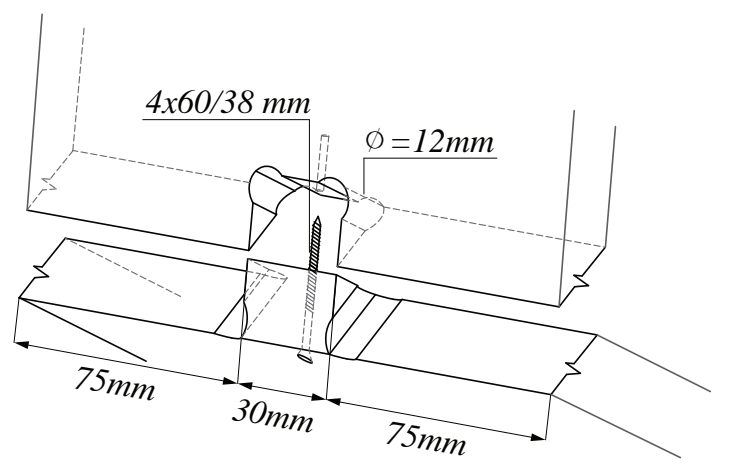

Figure 8: One-faced finger joint with a screw for applying pressure. Joint spacing along the edge was taken as $180 \mathrm{~mm}$; in this figure pressure area of only one screwed joint is shown. surface area. This enabled for all the discrete loads to be of equal size as well. Additional issues arose from the fact that in bidirectional folded surface structures individual plates lie in different planes, so the direction and the amount of the plate displacement varies depending on its position in a global assembly. As a solution, a load application setup was devised, which enables simultaneous, continuous loading of discrete plates while compensating for their differential displacements (Fig. 9). A system of pulleys with a steel wire running through, was positioned at each of the three longitudinal lines of loaded elements and the displacement-controlled load was introduced at the end of each system (see Fig9 9 ). Loading system energy losses due to friction and other causes were not taken into account in the performed experiments. Fitting a cubic polynomial regression model to the preliminary test data, showed that the forces exerted onto each plate during the course of the experiment resulted in a coefficient of determination equal to 0,99 . This confirmed that the uniform load was well approximated and that all the point loads applied on the structure could be considered equal over time.

Boundary conditions that allow rotation about $-y$ axis were used along the two supporting sides (see Fig $9 \mathrm{~b}$ ). The structure was inserted into the $18 \mathrm{~mm}$ deep slots in the timber part of the supports, and fixed using additional timber slats and self-tapping screws placed crosswise.

\subsection{Instrumentation and Loading Procedure}

Force transducers, LVDT's and inclinometers were placed 372 and marked as shown in Fig.(9 $)$. Additionally, a threedimensional digital image correlation (DIC) system was used for obtaining strain and deformation fields of the entire structure. A set of cameras was placed on a cantilever above the setup for securing a clear view of the entire structure's top surface. The observed surface was painted white after which a random speckle pattern was applied for allowing the analysis software to easily track the deformation to sub-pixel accuracy. Loading procedure was established according to 21. The load was applied in a quasi-static 
(a)
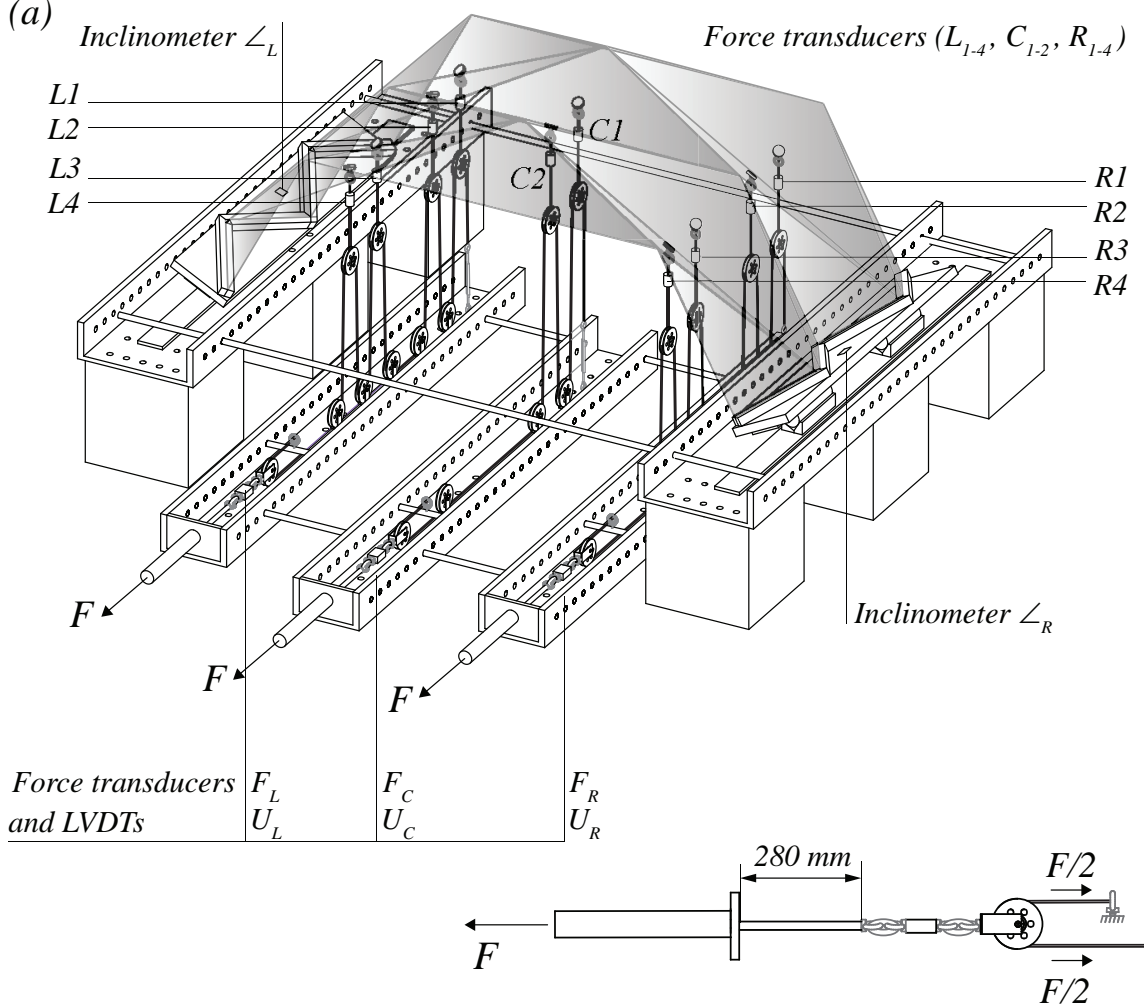

(b)

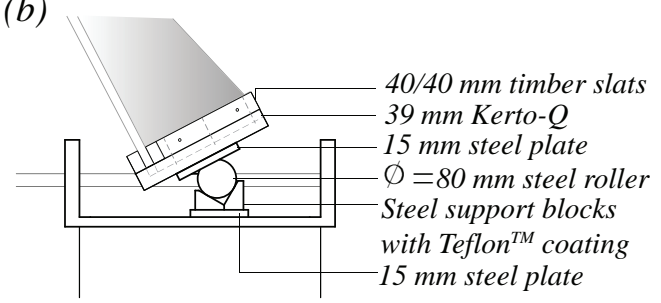

(c)

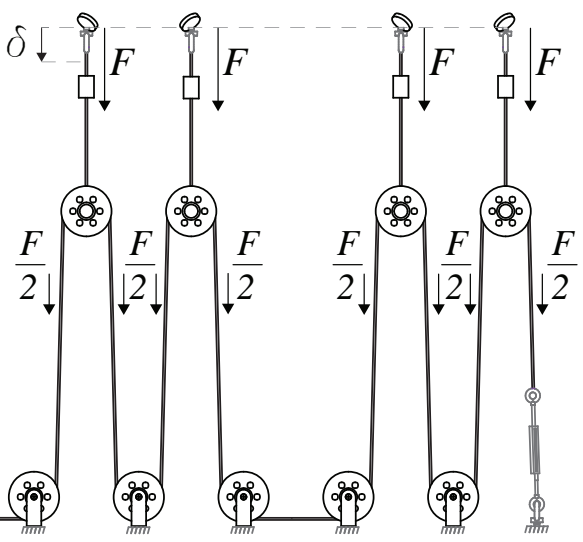

rate using a combination of displacement control and load control methods. For a detailed description of the used instrumentation and the test procedure the reader is referred to appendix B.

\section{Results}

Total load vs. midspan displacement curves of three types of tested structures are shown in (Fig 10$)$. Maximum displacements corresponding to the applied loads were obtained from the DIC system at points as shown in (Fig 11). Each of the three groups consisted of three experimental replicates. The results show that the highest structural stiffness is achieved when using adhesive joints, $55 \%$ higher than MTSJ open slot and 26\% higher than MTSJ closed slot structure elastic range stiffness. (see Table 22. For all three structure types the results suggest that serviceability limit state (SLS) would be the one to govern the design of timber folded surface structures. For a span of $2,9 m$ the SLS maximal allowed dis- placement, equal to $9,66 \mathrm{~mm}$ ( $L / 300$ according to [13]), stays well within the elastic stage for all tested structures (Fig 10p). The influence of the joint semi-rigidity on the displacements distribution and corresponding maximum value position is clearly visible in Fig 11 For the least rigid connections, i.e. open slot MTSJ, the maximum values of displacements occur at the edge (Fig 11p). As the rigidity of the joint increases for closed slot MTSJ, the distribution of displacements changes, locating the maximum value in the center of the two mid plates, around the loading ring (Fig 11 k). The most rigid case of adhesively joined structures retains the same position of the maximum displacement value as closed slot MTSJ, however the distribution demonstrates higher concentration around the loading ring $(\operatorname{Fig}[11$ a). The ratio of plate center over mid edge displacements at points marked $\mathbf{x}$ for adhesively joined, MTSJ open and closed slot structures is equal to 1.22, 0.97 and 1.07 respectively. Furthermore, even though the adhesively joined structures exhibited higher stiffness 
when compared to MTSJ closed slot structures, their characteristic total load at elastic limit point as well as mean maximum strength values are lower, respectively $14.5 \%$ and $21.26 \%$ lower than for MTSJ closed slot (see Table2). All three structure types failed as a result of connection failure, however, the failure mode and its progression were distinct for each type and are therefore explained in the following text (see Fig,12).

In adhesively joined structures, the first crack appears when the tensile stresses at the edge interface surpass the adhesives yield strength in tension (see point $\mathbf{A}$ in Fig 12 ). Opening of subsequent cracks and widening of existing ones follows shortly after, causing progressive reduction in stiffness (see point B). For replicates 2 and 3, after reaching its maximum load-bearing potential, point (C) marks the sudden load drop associated with brittle connection failure along the full edge length simultaneously. Subsequently, as folded surface structures form statically indeterminate systems, a redistribution of forces within the system follows. Finally, when the alternative load paths become overloaded, multiple edges fail simultaneously in tension, (see point D). Unlike replicates 2 and 3, replicate 1 shows higher initial stiffness and maximum achieved load, but consecutive brittle failure at points $(\mathbf{C})$ and $(\mathbf{D})$ follow with almost no force redistribution in-between. For all three replicates after point $(\mathbf{D})$ complete collapse follows in form of loss of structural integrity. It can be seen that failure happens due to tension entirely within the glued interface, where timber plates experience no structural damage (Fig 13).

For three replicates of structures with MTSJ open slots (see Fig 12 p), (A) marks a point after which reduction in the slope of the graph occurs, i.e. end of linear region, the gap caused by the slip of the joints at that moment is shown at the respective photograph. However, due to joint geometry defining the inclination of the tabs and slots side faces, the increase of the gap between the respective edges stops at a certain moment, i.e. when the gap size reached about $1 / 3$ of the plate thickness, and does not lead to complete edge separation. Instead, as the load increases most of tabs and slots side faces lock in contact and disable the further gap growth, the further transfer of bending moments is then enabled through compression of the side faces surfaces, therefore providing additional load bearing capacity to the structure. This further causes tension perpendicular to the plate plane and finally material failure by delamination (Fig 14 a), resulting in full loss of contact along one entire edge, (see point $\mathbf{B}$ in Fig $12 \mathrm{~b}$ ). The second critical edge of the two half-sized side plates fails at point $(\mathbf{C})$. In replicate 1 these two events happen simultaneously, characterized by a significant sudden load drop. At this point the two respective plates are no longer part of the load bearing system and their failure causes a rotation of the near-by side support (shown on the example of MTSJ closed slots replicate 3 in Fig 15). This further results in failure propagation in other edges. Due to large displacements, where maximum piston stroke was exceeded, the tests were stopped at point $(\mathbf{D})$. However, the structural integrity of main load-bearing elements was still preserved at this point. This suggests that the replicates would continue to sustain load as far as the connections between the main loaded plates would facilitate it.

In addition to the chosen MTSJ open slot theta angle combination, an example is also shown of a structure where all joint insertion vectors were chosen to be parallel to the $-y$ axis. This resulted in two different sets of angles, $\theta_{1}=0^{\circ}, \theta_{2}=27^{\circ}, \theta_{3}=20^{\circ}$ for the skewed edges, and $\theta_{1}=0^{\circ}, \theta_{2}=0^{\circ}, \theta_{3}=20^{\circ}$ for edges parallel to $-x$ axis. In fact, this was the initial set of angles intended for MTSJ open slot structures. However, after the structure with this set of angles showed deficiencies in mechanical behaviour within the global assembly, the final choice was modified. Significant edge opening was already observed at point (A) at a very low total load of $4.7 \mathrm{kN}$, when the first slip between adjacent tab and slots occurred (see MTSJ open slot $\left(\theta_{1}=0^{\circ}, \theta_{2}=27^{\circ} / 0^{\circ}, \theta_{3}=20^{\circ}\right.$ ) 


\begin{tabular}{llll}
\hline & Adhesively joined & MTSJ open slot & MTSJ closed slot \\
\hline Characteristic linear region stiffness $[\mathrm{kN} / \mathrm{mm}]$ & 3.67 & 1.65 & 2.69 \\
Characteristic total load when reaching SLS $[\mathrm{kN}]$ & 35.44 & 15.97 & 25.99 \\
Characteristic total load at elastic limit point $[\mathrm{kN}]$ & 58.51 & 38.46 & 68.44 \\
Mean maximum achieved strength $[\mathrm{kN}]$ & 79.45 & 61.86 & 100.91 \\
\hline
\end{tabular}

Table 2: Comparison of results of three different large scale structure types.
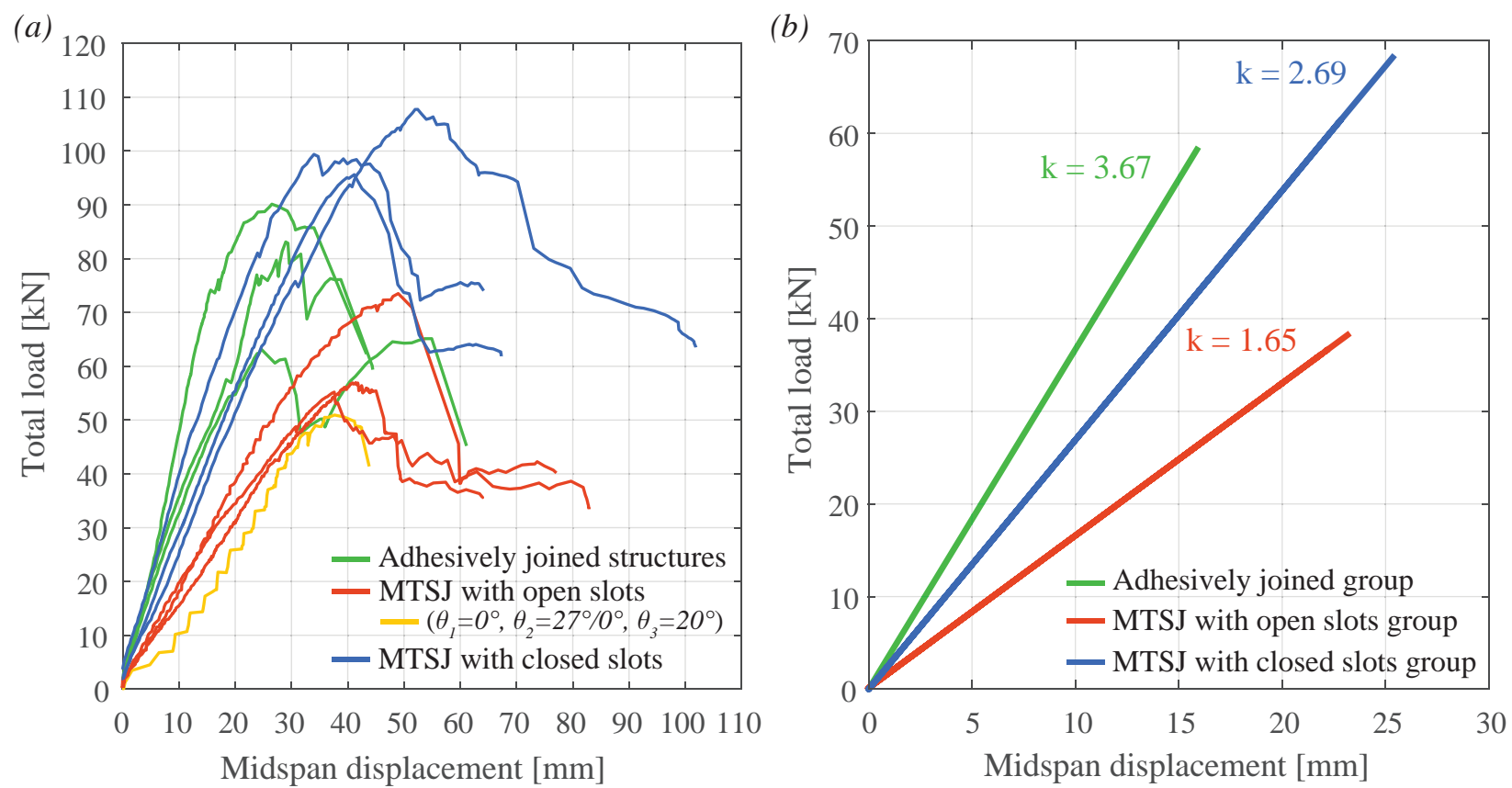

Figure 10: (a) Total load vs. midspan displacement. Triplets of curves of identical connection type are marked with the same color. The part of the curves corresponding to the unloading of the specimens according to the established loading protocol are omitted for clarity; (b) Characteristic elastic region and stiffness, $k$, for each respective group. The coefficient $k$ was determined by fitting a linear regression model to each of the 3 groups containing 3 replicates. The upper bound of the elastic region for each replicate was determined by imposing $R^{2}>0.99$, which corresponded to approximately $0.6 F_{\max }$ for all replicates.

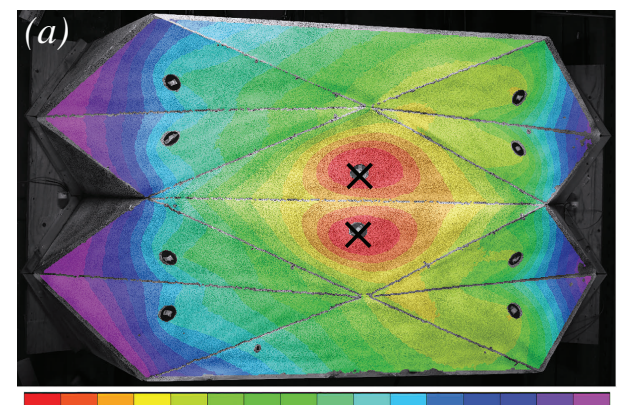

$-6.65$
$\mathrm{W}[\mathrm{mm}]$

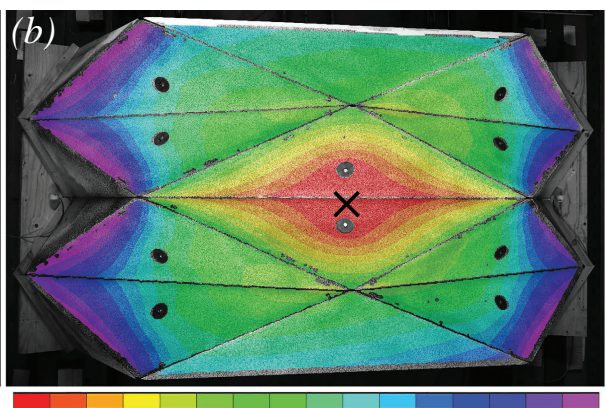

$-1,2-15.55$

$\mathrm{W}[\mathrm{mm}]$

$-0,1-10.60$

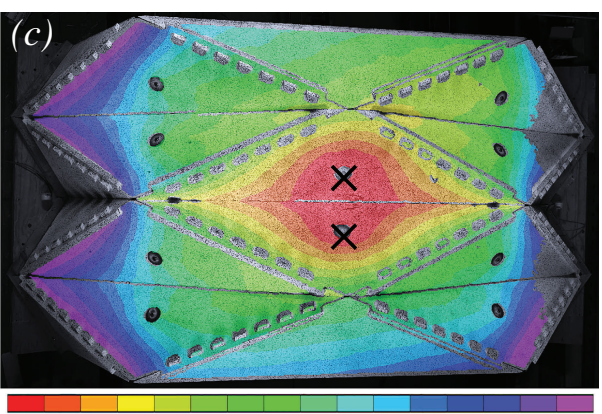

$\mathrm{W}[\mathrm{mm}]$

$-0,6$

Figure 11: Displacement fields of each of the tested structure type unfavorable replicate, shown at the moment when the total load on the structure amounted to $25 k N$; (a) Adhesively joined structure, replicate 3; (b) MTSJ with open slots, replicate 3; (c) MTSJ with closed slots, replicate 3. Points at which the displacement data was extracted for each type are marked with $\mathbf{x}$, in case of (a) and (c) as the maximum displacement found around the loading ring. 
(a) Adhesively joined structures

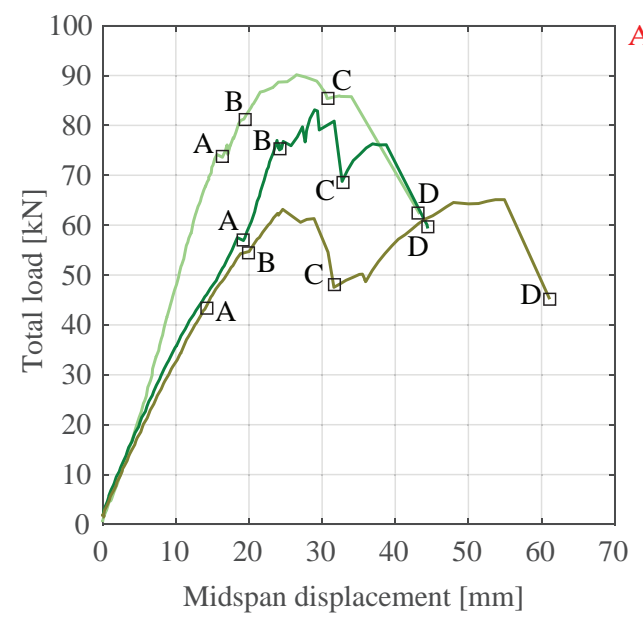

(b) MTSJ with open slots

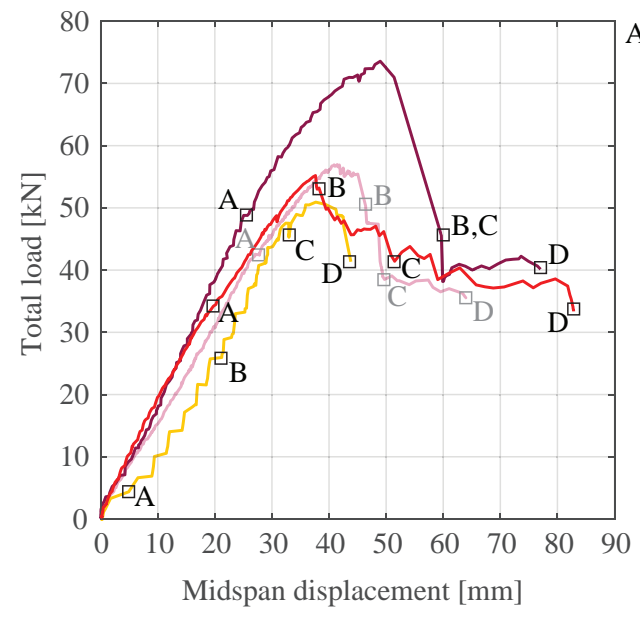

(c) MTSJ with closed slots

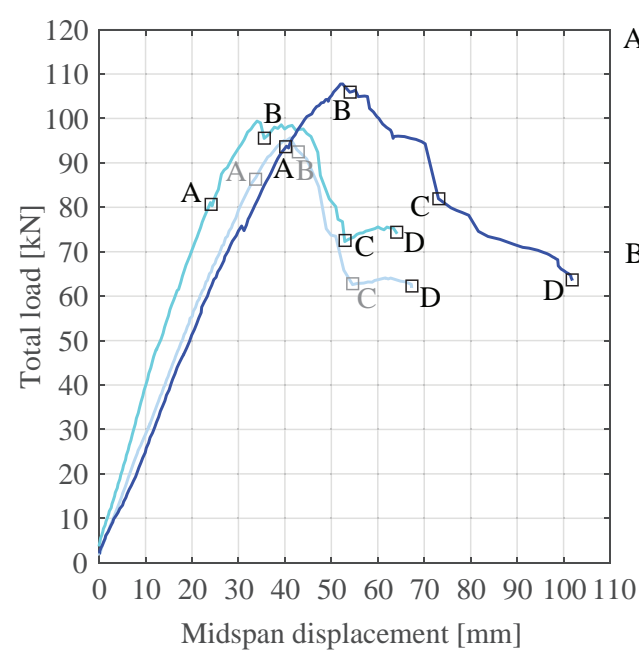

— Replicate 1

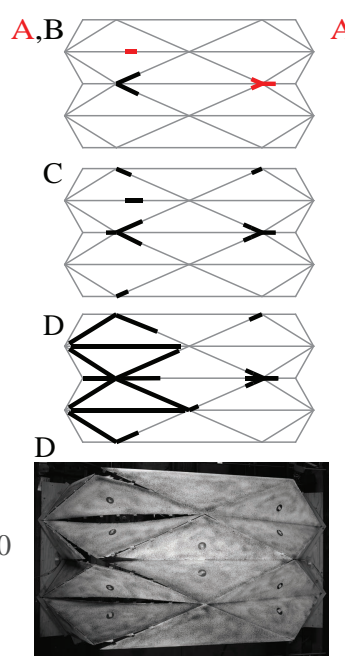

$$
\text { — Replicate } 1
$$

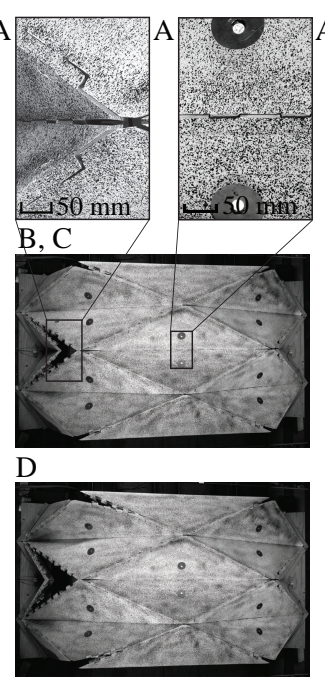

*(typology of failure same as in replicate 2)

\section{- Replicate 1}
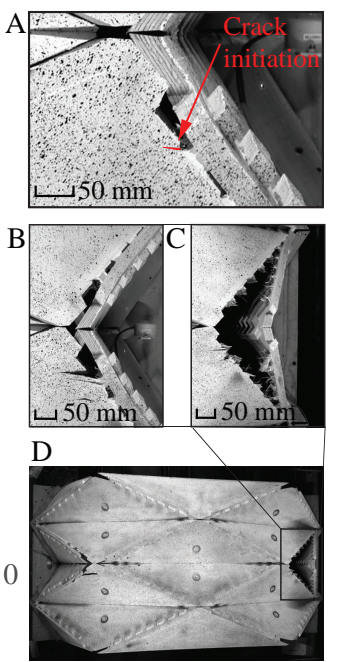

*(typology of failure same as in replicate 1)

- Replicate 2

- Replicate 2

Tab failure
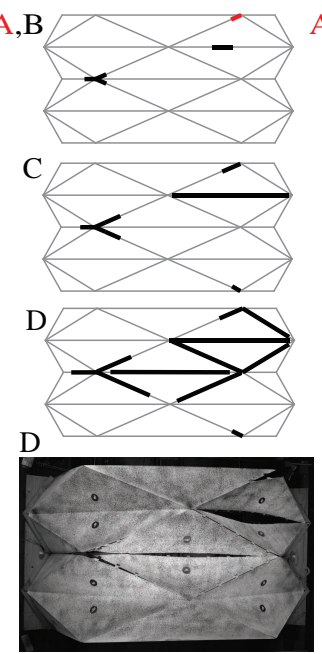

$\quad\left(\theta_{1}=0^{\circ}, \theta_{2}=27^{\circ} / 0^{\circ}, \theta_{3}=20^{\circ}\right)$

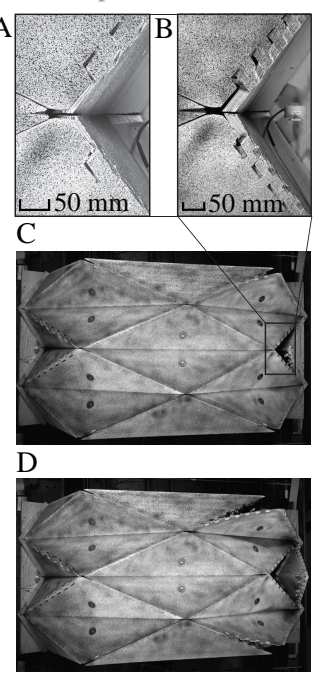

- Replicate $2 *$
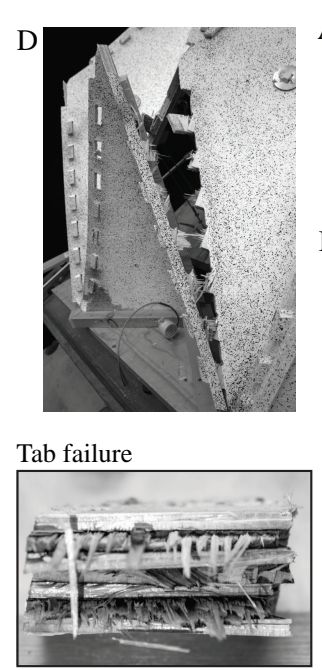

- Replicate 3

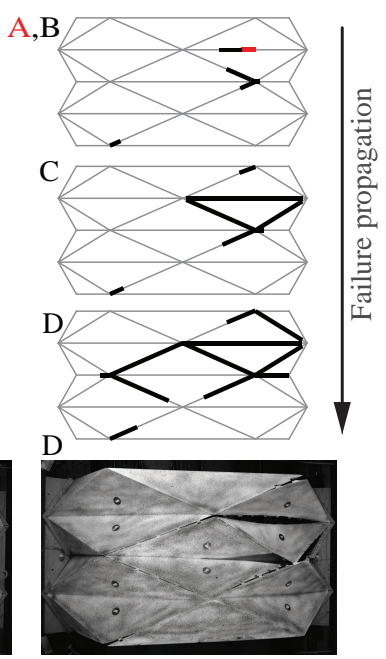

- MTSJ open slot

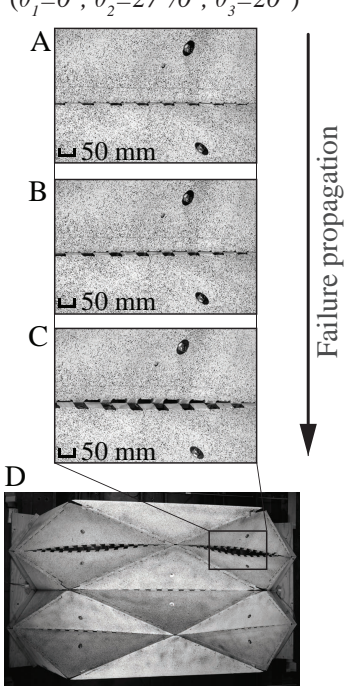

- Replicate 3

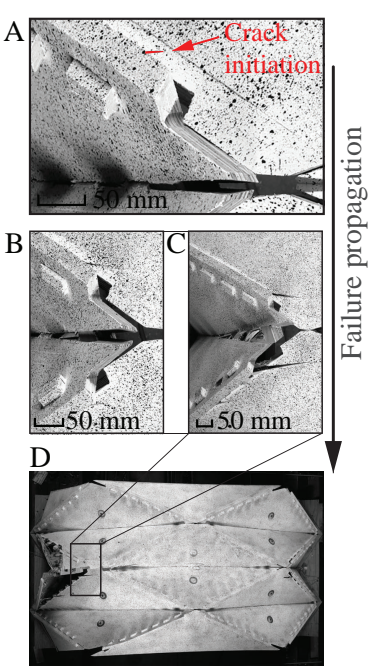

Figure 12: Individual total load vs. midspan displacement graphs and failure propagation for each of the tested structure type. 


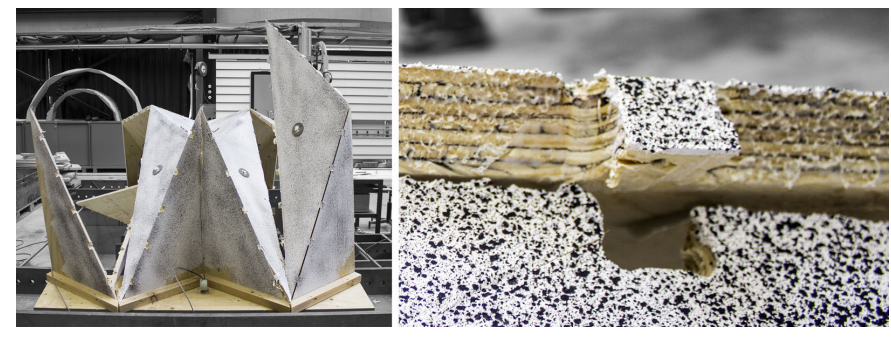

Figure 13: Loss of structural integrity in adhesively joined structures after point (D)(left) and detail of edge after failure (right); Replicate 3 is shown as a representative of all three tested replicates since they exhibited same type of final collapse.
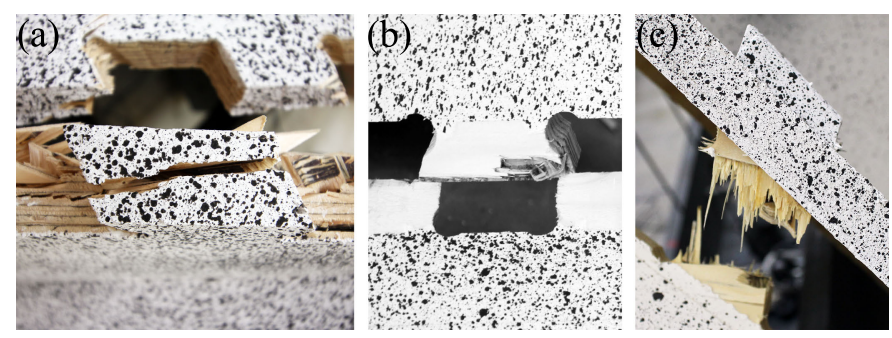

Figure 14: MTSJ failure modes; (a) open slot; (b) open slot $\left(\theta_{1}=0^{\circ}\right.$, $\theta_{2}=27^{\circ} / 0^{\circ}, \theta_{3}=20^{\circ}$ ); (c) closed slot. much steeper initial slope of the graphs in comparison to the ones with open slots, indicating a higher stiffness. This constant slope is followed by a kink, corresponding to the initiation of first crack visible at the top surface which appears at the point marked as (A). It can be seen that this localized event hardly influences the global structure stiffness. Structural failure occurs and softening begins when material capacity in tension perpendicular to the plate main grain orientation is exhausted at the connection level (Fig 14c). At that point, the first tab closest to the crack fails, (B), characterized by the abrupt increase in the respective support rotation (Fig 15$)$. This greatly contributes to further enhancement of the tensile forces occurring at the long skewed edges of the half-sized plates. With continuation of imposing displacements, support rotation continues and failure progresses to the next tab and so on, until the end of the edge is met at the supports, (C). Same as in MTSJ with open slots, the tests were stopped at point (D). However, at this point cracks along the midThey were caused by out-of-plane bending when material capacity in tension perpendicular to the plate grain orientation was exceeded, this time at panel level. Fracture at this level was observed only in MTSJ with closed slots, as opposed to other tested structure types, where it was always constrained to the level of connections.

\section{Discussion}

Comparing large scale to the preliminary detail test results, it is noted that the failure modes of connections within large scale structures are very similar to the ones observed in small scale samples under bending. They are altered mainly by additional tension forces which appear at the edges perpendicular to the panel main grain orientation, i.e. global structure $-y$ axis direction. Certainly, due to complex geometry, where discrete plates lie in different planes, the failure modes in large scale replicate connections are additionally influenced by twisting moments and dle of loaded plates bottom face were observed (Fig 16). 


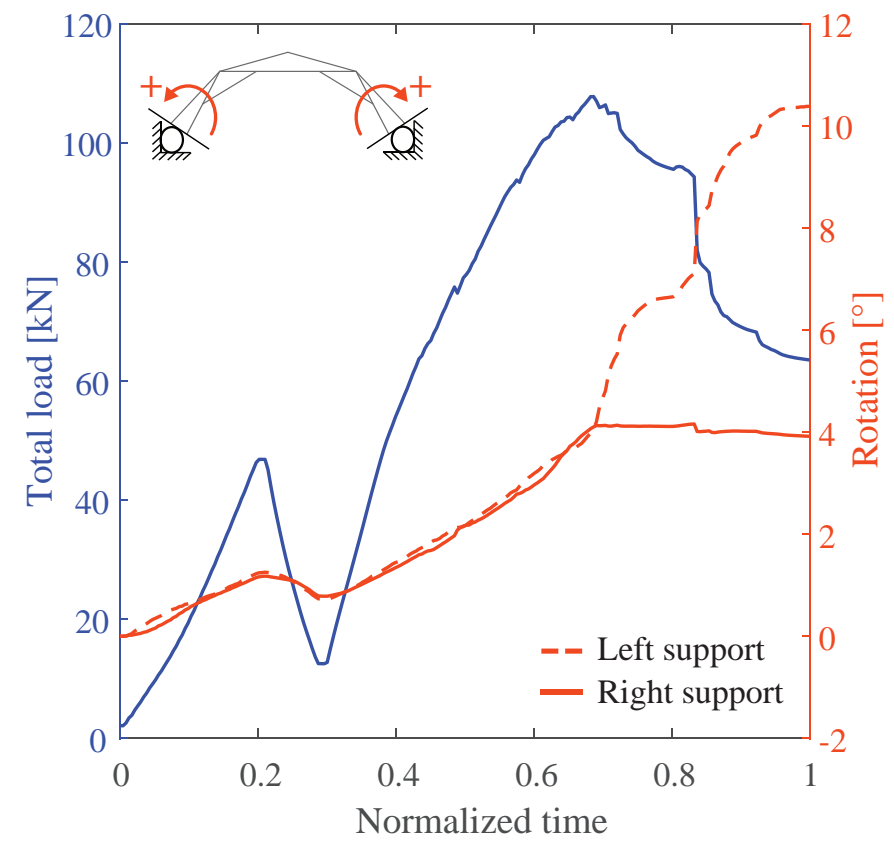

Figure 15: Total load and rotation of supports vs. normalized time shown on the example of MTSJ open slot replicate 3. Sudden increase in rotation of left support occurs at the moment of MTSJ failure at the left side connection between half and full-sized plate. Both MTSJ open and closed slot structure replicates experienced the same reaction, where the support side with rotation increase varied depending on the failure side. other edge forces, mainly shear, as well as joint edges not being parallel to the main plate grain orientation. However, the similarity still suggests that, together with tension perpendicular to grain which is a known weakness of timber, bending around the edges is one of the most critical loading conditions when discussing timber folded surface structures. Furthermore, when looking at the initial slip of MTSJ open and closed preliminary detail test and comparing it to the respective MTSJ large scale tests, an important influence provided by interlocking is noticed. The initial slip effect in a multiple plate assembly is completely eliminated, mutual blocking of neighbouring edges is achieved as soon as the structure is put in position. This outcome is most visible in structures with MTSJ open slots.

MTSJ structures, both open and closed, experienced similar type of failure. For all six replicates it occurred at

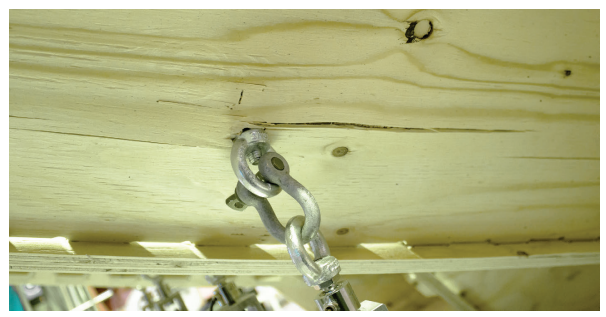

Figure 16: MTSJ with closed slots, replicate 3. Failure along the middle of the loaded plate bottom face.

either the left or the right side at mid length of the structure, when the two half-sized plates edges failed in tension. As explained in [12, structural behaviour of folded surface systems is defined by a mixture of extensional and flexural plate actions. Locally, at individual plate level the applied load is transferred to the plate edges by out-of-plane bending, where it is then resolved into components lying in planes of the adjoining plates. These are transferred between two adjacent edges by compression, when a pair of joint interfaces come into contact. In MTSJ with open slots there are three shared joint faces that can facilitate this transfer in compression, $i j_{1,2,3}\left(\mathrm{Fig} / \mathrm{17}_{\mathrm{a}}\right)$. Faces $i_{4}$ and $i_{5}$ remain "open" and have no contact pair, resistance to the movement in their direction is achieved only through inclination of faces $i j_{1}$ and $i j_{3}$ when adequate Bryant angles are used (friction between the contact pair faces is not taken into consideration here). The mentioned faces lack of contact pair causes slipping when partial or complete loss of contact in compression occurs due to bending around the edges. Contact loss is additionally enhanced by inevitable fabrication tolerances and possible imperfections. On the other hand, in MTSJ with closed slots four pairs of contact faces exist, $i j_{1,2,3,4}$ (Fig $17 \mathrm{~b}$ ), increasing its load-bearing potential. In this case, slip is only possible in the direction of insertion vector, so its influence is greatly reduced. The above explained difference between the MTSJ open and closed slot is indicated by the smoothness of their total load vs. midspan displacement curves, Fig 10 .

Additionally, MTSJ open slot structures exhibited rela- 
tively large edge openings quite early in their elastic stage, at $4.7 \mathrm{kN}$ load the gap between the two mid plates amounted to $8.75 \mathrm{~mm}$ which is equal to $41 \%$ of plate thickness, as well as abrupt slipping between edges during testing, even after correcting the initial set of Bryant angles intended for these structures. This indicates that they cannot be considered reliable for providing efficient load transfer between plates in large assemblies. In such structures plates and their edges lie in different planes, and even though this is what contributes to such joints mutual interlocking ability, it also implies forces acting in various directions. Accordingly, in order to avoid the undesirable behaviour mentioned above, the set of Bryant angles describing the joint geometry would have to be determined for each edge separately, depending on the respective load direction. Even so, there would be no way of ensuring they could retain their capacity for changing load conditions.

With respect to adhesively bonded structures, the semirigid behaviour of MTSJ with closed slots connections provides additional contribution to the system ductility. In such structures the cause of ductility after yielding as well as failure is twofold. Firstly, plastic behaviour after the yield point is enabled by the ductility of the connections. Even though timber is generally considered to be a brittle material, especially in tension, it does provide a substantial level of ductility in compression. This is very effectively utilized by MTSJ with closed slots as they transfer all edge occurring forces through compression between their adjoining faces. Such semi-rigid behaviour of connections is considered to be beneficial for increasing the reliability of the global system. [22, 23, 24]. Secondly, global structural ductility is achieved through redundancy of load paths. Due to their topology, folded surface structures form statically indeterminate systems, where the redistribution of forces between elements follows after their individual failure. The second is however also true for the tested adhesively bonded structures. But in their case, as the connections are very rigid and fail along the entire

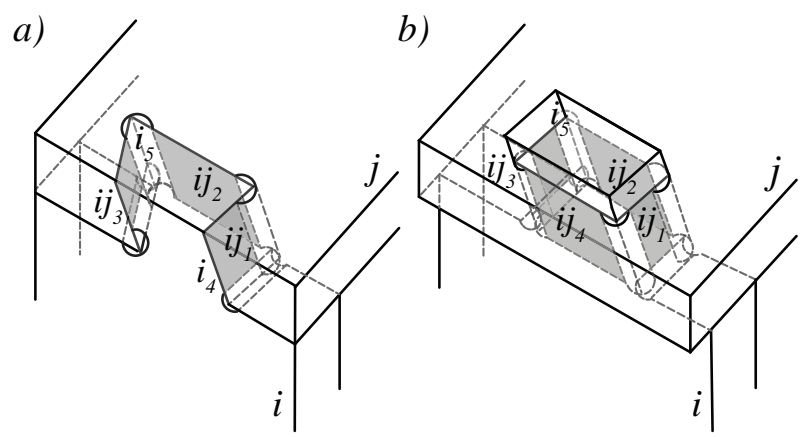

Figure 17: MTSJ contact faces pairs; (a) open slot; (b) closed slot.

edge length simultaneously in a brittle manner, the soon ${ }_{638}$ following system failure is brittle as well. So in this case, ${ }_{639}$ it is the topology alone that provides the redistribution of 640 forces and avoidance of complete structural failure immediately after the adhesive ultimate strength is achieved.

Although adhesively bonded connections generally provided the highest stiffness of the structure, they have multiple disadvantages for building scale applications. In-situ gluing of structural joints is not recommended and it is usually preformed off-site where constant curing conditions can be maintained. This implies preassembly and the loss of flat packing advantage of folded surface systems made of multiple discrete elements. When using adhesives, the moisture content of the components must be controlled and adequate pressure without relative movement has to be assured. This proved to be difficult even in controlled laboratory conditions. The aligning of the plate edges was aided by introducing one-faced finger joints, however they could not ensure a precise dihedral angle positioning. The build-up of the so caused small inaccuracies in individual assembly, later caused gaps when positioning the structure on supports. In the presented case the gaps were small enough $(\max 25 \mathrm{~mm})$ to be closed by the timber slats used for fixing the structure on supports. However in larger structures, inaccuracies of such scale could cause more significant problems.

Considering the feasibility of tested structures types for building scale, they are further compared in terms of fab- 
rication time and assembly (Table 3). Global and connection geometry generation for each structure was very fast and easy with the use of the developed tool (Appendix A). The tool also enabled the direct output of the CNC G-code used for fabrication. The fabrication time depends on the contour length and number of vertices, i.e. corner points, of each plate. It is a function of the CNC machine type, its maximum speed and number of used axis. In the presented case, 5-axis MAKA MM7S CNC machine was used with a target cutting speed of $5000 \mathrm{~mm} / \mathrm{min}$. The total fabrication time shown in (Table 3 ) consists of preparation time needed to position and remove the cut panels from the machine as well as machining time itself. Machining includes: pre-drilling of holes for screws used for fixing the panels, drilling the holes at loaded plates geometrical center for fixing the pulleys, engraving plate numbers for later assembly, planarizing panel surface for achieving constant thickness and finally, cutting of the plate edges with integrated connectors. Plate edges were cut in 2 passes of the milling tool. All except the final machining step took equal amount of time for all three structure types, $\sim 120 \mathrm{~min}$. The biggest difference was noted in the assembly time, where adhesively joined structures proved to be quite time consuming. Two people were needed for holding the adjacent plates in position while the third one was necessary for spreading glue along the edges and inserting screws for applying pressure. The MTSJ structures, on the other hand, were very easy to assemble and only 2 people were required. Within the time noted in (Table 3 the time for curing of the adhesive is not included $\sim 45 \mathrm{~min}$, as well as the $2 \mathrm{~h}$ required after the curing before further processing of the replicates [25]. It is important to note that all of the above mentioned times reflect the laboratory resources and conditions in which the replicates were produced.

\section{Conclusions}

In this paper, structural behaviour of timber folded surface structures was observed under continuous load and the influence of three different connection types was studied. Thereby, not only the global load-displacement behaviour was analysed, but also the occurring failure modes, based on detailed photo documentation of failure propagation obtained from the DIC system. Based on the obtained results and observations, the conclusions are as follows:

- When reaching the the maximal SLS prescribed displacement, all three tested structure types stay well within their elastic stage, exhibiting high reserve of load bearing capacity. However, the presented large variation in the elastic range stiffness of structures with different connection details, demonstrates the importance of taking the MTSJ semi-rigid behaviour into consideration in future evaluations of timber folded surface systems for structural application.

- All tested structures failed by exceeding the connection detail load bearing capacity. In addition to tension perpendicular to grain as the main cause of failure, the similarity between large scale and preliminary detail bending test failure modes indicates that bending is also one of the crucial loading cases when considering integrally attached timber folded surface structures.

- MTSJ open slot structures large scale test results indicate that such structures cannot be considered reliable for providing efficient load transfer between plates in large assemblies.

- Although, adhesively bonded connections provide higher ${ }_{31}$ stiffness when compared to MTSJ structures, due ${ }_{732}$ to multiple disadvantages considering the use of ad- ${ }_{733}$ hesives, their application for building scale timber ${ }_{734}$ folded structures suggests to be unfeasible. 


\begin{tabular}{llll}
\hline & Adhesively joined & MTSJ open slot & MTSJ closed slot \\
\hline Contour length / No. of vertices & $134,44 \mathrm{~m} / 2408$ & $171,15 \mathrm{~m} / 3834$ & $224,46 \mathrm{~m} / 4398$ \\
Total fabrication time (ca.) & $3: 00 \mathrm{~h}$ & $3: 30 \mathrm{~h}$ & $3: 50 \mathrm{~h}$ \\
Assembly time (ca.) / No. of people & $3 \mathrm{~h} / 3$ & $1 \mathrm{~h} / 2$ & $1 \mathrm{~h} / 2$ \\
\hline
\end{tabular}

Table 3: Fabrication and assembly time for each of the tested large scale structure type.

- MTSJ with closed slots structures demonstrated the highest load-bearing potential leading to a structural efficiency of 158.3 , i.e. strength-to-weight ratio. $21 \%$ and $38 \%$ higher than the characteristic structural efficiency of adhesively joined and open slot MTSJ structure respectively.

- The MTSJ with closed slot connection semi-rigidity provides additional ductility to the system, making such connections highly beneficial concerning the ultimate load-bearing capacity as well as the stiffness of the structures in the elastic range. In this manner robust structural systems with residual resistance are obtained, where localised failure does not endanger global structure integrity.

\section{References}

[1] A. Lebee, From folds to structures, a review, International Journal of Space Structures 30 (2) (2015) 55-74.

[2] E. Onate, Structural Analysis with the Finite Element Method. Linear Statics, 1st Edition, Vol. 2. Beams, plates and shells of Lecture Notes on Numerical Methods in Engineering and Sciences, Springer, 2013.

[3] M. Simek, V. Sebera, Traditional furniture joinery from the point of view of advanced technologies, in: Proceedings of the International Convention of Society of Wood Science and Technology and United Nations Economic Commission for Europe, Geneva, Switzerland, 2010.

[4] J. Knippers, et al., From nature to fabrication: Biomimetic design principles for the production of complex spatial structures, in: L. Hesselgren, S. Sharma, J. Wallner, N. Baldassini, P. Bompas, J. Raynaud (Eds.), Advances in Architectural Geometry 2012, Springer Verlag, 2013.
[5] C. Robeller, S. Nabaei, Y. Weinand, Design and fabrication of robot-manufactured joints for a curved-folded thin-shell structure made from clt, Robotic Fabrication in Architecture, Art and Design (2014) 67-81.

[6] C. Robeller, A. Stitic, P. Mayencourt, Y. Weinand, Interlocking folded plate - integrated mechanical attachment for structural timber panels, in: P. Block, J. Knippers, N. J. Mitra, W. Wang (Eds.), Advances in Architectural Geometry 2014, Vol. 4 of Advances in Architectural Geometry, Springer Verlag, 2014, pp. 281-294.

[7] S. Roche, G. Mattoni, Y. Weinand, Rotational stiffness at ridges of timber folded-plate structures, International Journal of Space Structures 30 (2) (2015) 153-167.

[8] M. Dedijer, S. Roche, Y. Weinand, Shear resistance and failure modes of edgewise multiple tab-and-slot joint (mtsj) connection with dovetail design for thin lvl spruce plywood kerto-q panels, in: J. Eberhardsteiner, W. Winter, A. Fadai, M. Poll (Eds.), Proceedings of the World Conference of Timber Engineering, TU Verlag, 2016, pp. 1516-1523.

[9] P. Haller (Ed.), COST C1: Semi-Rigid Timber Joints- Structural Behaviour, Modelling and New Technologies, Semi-Rigid Behaviour of Connections in Civil Engineering, COST, European Cooperation in the field of Scientific and Technical Reserach, 1992-1999.

[10] C. C. Baniotopoulos, F. Wald (Eds.), The Paramount Role of Joints into the Reliable Response of Structures: From the Classic Pinned and Rigid Joints to the Notion of Semi-rigidity, Vol. 4 of NATO Science Series II: Mathematics, Physics and Chemistry, Springer Netherlands, 2000.

[11] VTT Technical Research Centre of Finland, certification body (S017, EN 45011) accredited by FINAS, Certificate for structural laminated veneer lumber, no. 184/03 (2009).

[12] A. Stitic, Y. Weinand, Timber folded plate structures topological and structural considerations, International Journal of Space Structures 30 (2) (2015) 169-177.

[13] European Committee for Standardization, Brussels, EN 19951-1, Eurocode 5: design of timber structures - part 1-1: generalcommon rules and rules for buildings (2004).

[14] S. Roche, J. Gamerro, Y. Weinand, Multiple tab-and-slot joint: 
improvement of the rotational stiffness for the connection of thin structural wood panels, in: J. Eberhardsteiner, W. Winter, A. Fadai, M. Poll (Eds.), Proceedings of the World Conference of Timber Engineering, TU Verlag, 2016, pp. 1556-1564.

[15] RhinoCommon SDK for Rhino, https://github.com/mcneel/ rhinocommon accessed: 05-01-2017.

[16] S. Roche, C. Robeller, H. Laurent, Y. Weinand, On the semirigidity of dovetail joint for the joinery of lvl panels, European Journal of Wood and Wood Products 73 (5) (2015) 667-675.

[17] P. Huybers, See-through structuring. a method of construction for large span plastic roofs, Ph.D. thesis, TU Delft (1972).

[18] H. Buri, Origami - folded plate structures, Ph.D. thesis, Ecole Polytechnique Federale de Lausanne (2010).

[19] H. Hossdorf, Model analysis of structures, Van Nostrand Reinhold Company Ltd., 1974.

[20] H. Harris, G. Sabnis, Structural modelling and experimental techniques, 2nd Edition, CRC Press, 1999.

[21] ISO 6891:1983, Timber structures - Joints made with mechanical fasteners - General principles for the determination of strength and deformation characteristics (1983).

[22] P. H. Kirkegaard, J. D. Sorensen, D. Cizmar, V. Rajcic, System reliability of timber structures with ductile behaviour, Engineering Structures 33 (11) (2011) 3093-3098.

[23] A. Asiz, I. Smith, New generation of timber design practices and code provisions linking system and connection design, in: H. J. Larsen, J. Munch-Andersen (Eds.), CIB-W18 Timber Structures, A review of meetings 1-43, Part 2:Material properties, 2011, pp. 53-54.

[24] A. Jorissen, M. Fragiacomo, General notes on ductility in timber structures, Engineering Structures 33 (11) (2011) 2987-2997.

[25] Collano AG, Semparoc Rapid V- technical sheet, 2015, https://www.collano.com/downloads/datenblaetter/fr/ semparoc_rapid_v_fr.pdf, accessed: 20-01-2017.

[26] M. Botsch, et al., Polygon Mesh Processing, AK Peters, 2010.

[27] C. Robeller, Y. Weinand, A 3d cutting method for integral 1dof multiple-tab-and-slot joints for timber plates, using 5-axis cnc cutting technology, in: J. Eberhardsteiner, W. Winter, A. Fadai, M. Poll (Eds.), Proceedings of the World Conference of Timber Engineering, TU Verlag, 2016, pp. 2576-2584.

[28] M. Sutton, J. Orteu, H. Schreier, Image correlation for shape, motion and deformation measurements, Springer, 2010.

\section{Appendix A. Automatic Geometry Generation and Digital Fabrication}

The automatic generation of the $3 \mathrm{~d}$ geometry and the fabrication data allowed for the rapid creation of speci- men with variable geometry parameters, which would have been impossible with state-of-the-art CAD software tools. These functions were implemented through two custom developed programs, using the programming language Visual C\# and the Rhino Common Software Development Kit (SDK) [15]. A realtime preview of the output geometry was realized through the implementation as a CAD Addon for the visual programming software Grasshopper.

\section{Appendix A.1. Geometry Generation}

The generation of the plate geometry with MTSJ connectors is based on a target surface $S_{\text {target }}$. This surface is required in the form of planar facets, as a doublyconnected edge list (DCEL) data structure [26. This is a standardized structure for planar graphs, available in various software packages and software development kits. It allows for neighborhood request management, which is crucial for the program to generate the plate geometry. Additional input parameters are listed in table A.4. They include the width of the tenons $w_{\text {tenon }}$, the thickness of the plates $t_{\text {plate }}$, and a text string which sets the parameters for individual joints. This is possible through the identifiers of the edges in the polygon mesh data structure. Each of the edges is assigned a number, which is visualized by the program. Individual joint parameters for each of these edges can then be manually set by adding a line of comma separated values to the input string Jconfig. Each line sets the parameters for one edge, beginning with the identifier number of the edge (0) and the joint type (1). The next three values are used to set the X (2), Y (3) and Z (4) components of the joint insertion vector. If no custom configuration is specified for an edge, it is processed by default as a miter joint. The same applies to joints where the dihedral angle $\varphi$ lies outside of the possible range for

\section{DOF MTSJ.}

As its primary output, the program generates a pair of contour polylines for each of the plates. It consists of a top and bottom contour, which are joined together 


\begin{tabular}{llll}
\hline Input & \multicolumn{3}{l}{ Output } \\
\hline Parameter & Type & Parameter & Type \\
\hline$S_{\text {target }}$ & double & Plateconts 3d & Polylines \\
$w_{\text {tenon }}$ & double & Plateconts 2d & Polylines \\
$t_{\text {plate }}$ & double & Platebreps & BREPs \\
Jconfig & string & & \\
$L_{\text {trans }}$ & double & & \\
Flatten & bool & & \\
Breps & bool & & \\
\hline
\end{tabular}

Table A.4: MTSJ Generator Program.

from the polylines of the individual edges of each polygon facet. A transition segment is required at the start and end of each joint, towards the vertices of the polygons. The length of these segments is set through the input parameter $L_{\text {trans }}$, as a percentage of the edge length. Two additional, optional outputs can be generated through two boolean switches. The Flatten option will create a $2 \mathrm{~d}$ copy for each pair of plate contours, where the geometry is transformed from the $3 \mathrm{~d}$ plane of the target surface mesh facet it is based on, to the $2 \mathrm{~d}$ XY plane of the world coordinate system. This optional $2 \mathrm{~d}$ output is used for fabrication, where the plate contours need to be nested onto the timber plates. The BREPs option will create $3 \mathrm{~d}$ boundary representation elements (BREP) for each plate. These solids are collections of connected surface elements, based on the plate contour polygons. They are generated by the algorithm through a combination of two trimmed surfaces for the top and bottom contour polyline, and a loft surface in between the two contour polylines. The optional output of BREPs is needed for Finite Element based calculations and for visualization purposes.

\section{Appendix A.2. Fabrication Data Generation}

The Generation of the ISO6983 G-Code is created with a second program, which was custom developed for the MAKA mm7s 5-axis CNC router. This program generates the output string that contains the sequence of machin-

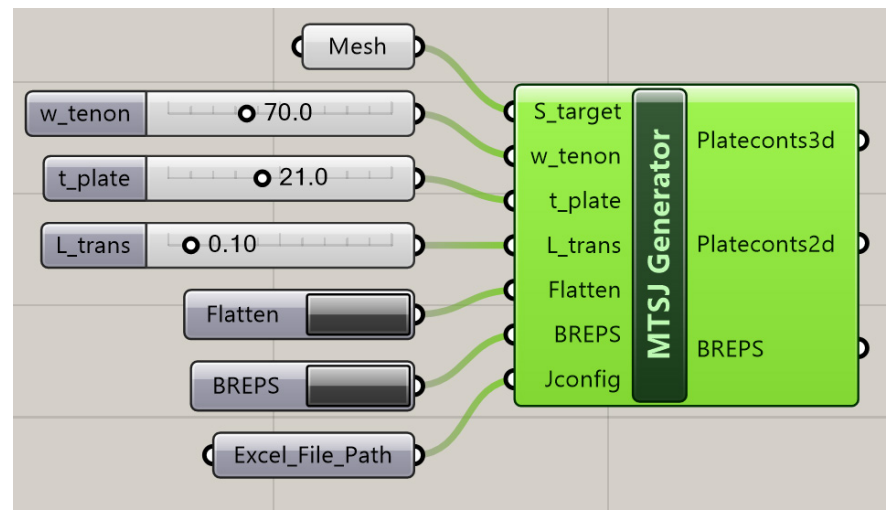

Figure A.18: MTSJ generator in Grasshopper.

ing instructions based on the Platecontour polyline pairs, which are created with the geometry generation program from section Appendix A.1. The number of segments in the top polyline and bottom polyline within a contour pair must be equal, as they define the quadrilateral polygon facets for the cutting. The triangular facets, which are required at the transition from MTSJ to miter joints, are generated as quadrilateral faces with two points at the same location. In the case of closed-slot MTSJ, a plate definition contains multiple pairs of top and bottom polylines. Each additional pairs defines a slot, which is differentiated from the primary pair of plate contours through an inverted orientation. While the orientation of outside contours is counter-clockwise, inside contours are oriented in a clockwise rotation. This is later reflected in the direction of the cutting tool path.

Finally, corresponding lists of consecutive tool path points and machining head cardan rotation angles in the output text string $G$-Code are calculated based on input parameters listed in table A.5. They include the tool radius $r_{\text {tool }}$, the number of vertical passes $n_{\text {infeed }}$, machine feed rates for the cutting velocity $F_{c u t t i n g}$ and $F_{a x i a l}$, and $\mathrm{Z}$ values for the definition of retreat and security planes, to which the tool moves during the fabrication. The input parameter Notches will automatically create tangential notches 27] on all concave corners, which is an essential part of the fabrication of integral timber plate joints. 


\begin{tabular}{llll}
\hline Input & \multicolumn{3}{l}{ Output } \\
\hline Parameter & Type & Parameter & Type \\
\hline Platecontours & double & G-Code & string \\
Z return & double & & \\
Z security & double & \\
$r_{\text {tool }}$ & double & \\
$n_{\text {infeed }}$ & integer & \\
$F_{\text {cutting }}$ & integer & \\
$F_{\text {axial }}$ & integer & \\
Notches & bool & \\
\hline
\end{tabular}

Table A.5: Plate Fabrication Program.

This feature can be deactivated for pre-passes, also called roughing, commonly used when machining thick plates.

\section{Appendix B. Test Instrumentation and Loading Procedure}

As marked in (Fig 9a the test instrumentation is as follows:

- $L_{1-4}, C_{1-2}, R_{1-4}$ : HBM U9C force transducers of $20 k N$ nominal force were placed above the pulleys at the centroid of each loaded plate.

- $U_{L}, U_{C}, U_{R}$ : Linear variable differential transformers (LVDTs) were positioned at the end of each cable for measuring the overall system displacements.

- $F_{L}, F_{C}, F_{R}$ : The total applied force is measured by HBM S9M force transducers of $20 k N$ nominal force, which are positioned at the hydraulic cylinders.

- $L_{L}, L_{R}$ : For controlling the rotation of the supports two AccuStar electronic inclinometers with a $\pm 60^{\circ}$ sensing range were fixed onto the slotted $39 \mathrm{~mm}$ timber plates.

Three-dimensional digital image correlation (DIC) system was used for obtaining strain and deformation fields of the entire structure. A set of two SVCam-HR29050 29
Megapixel GigE VISION cameras was fixed on a cantilever above the setup with the angle between the cameras equal to $21^{\circ}$ (Fig. B.19). The cameras were used with a Zeiss 35mm, f/8 Distagon ZF-I lenses and BP525 Green Bandpass Filters. The focal plane of the cameras was set at the bottom level of the two central plates fold. Even though the whole structure was within the cameras field of view, equal to approximately $3 \times 2 m$, some regions of the structure were difficult to capture. Particulary the half-size plates close to the supports, due to their high inclination with respect to the cameras position (Fig. B.20). The structure was symmetrically illuminated by two pairs of green LED Effilux light bars with a semi-opaque diffuser and a diffusion angle of $25^{\circ}$. The bars were mounted on the vertical steel columns. The speed of image acquisition was set at $0,1 \mathrm{~Hz}$ and the exposure time was equal to $35000 \mu$ s. DIC system control was performed by Correlated Solutions VIC 3D software.

The structure's top surface was painted matte white after which a random speckle pattern was applied with a pneumatic paint sprayer containing black paint (Fig. B.21). The calibration target of $12 \times 9-50 \mathrm{~mm}$ size and uniformly spaced markers was used. The structure shape easily facilitated the positioning of the target at different locations and various angles. It was important to keep the orientation of the target constant at all positions. Around two positions per plate at different angles were taken to obtain a good score after calibration (between 0,2 and 0,3 ) with order of distortion set to 2. Half-sized plates on the support sides were not included in the calibration process. The DIC system was calibrated for each test individually in order to ensure the accuracy of measured values. First, for every experiment five images were taken to test the reliability of the setup. Generally, the vertical displacement $V$ was considered the main indicator, and projection error values less then $0,01 \mathrm{~mm}$ were targeted when taking into consideration the entire area of interest. This area did not include the half-size plates close to the supports 


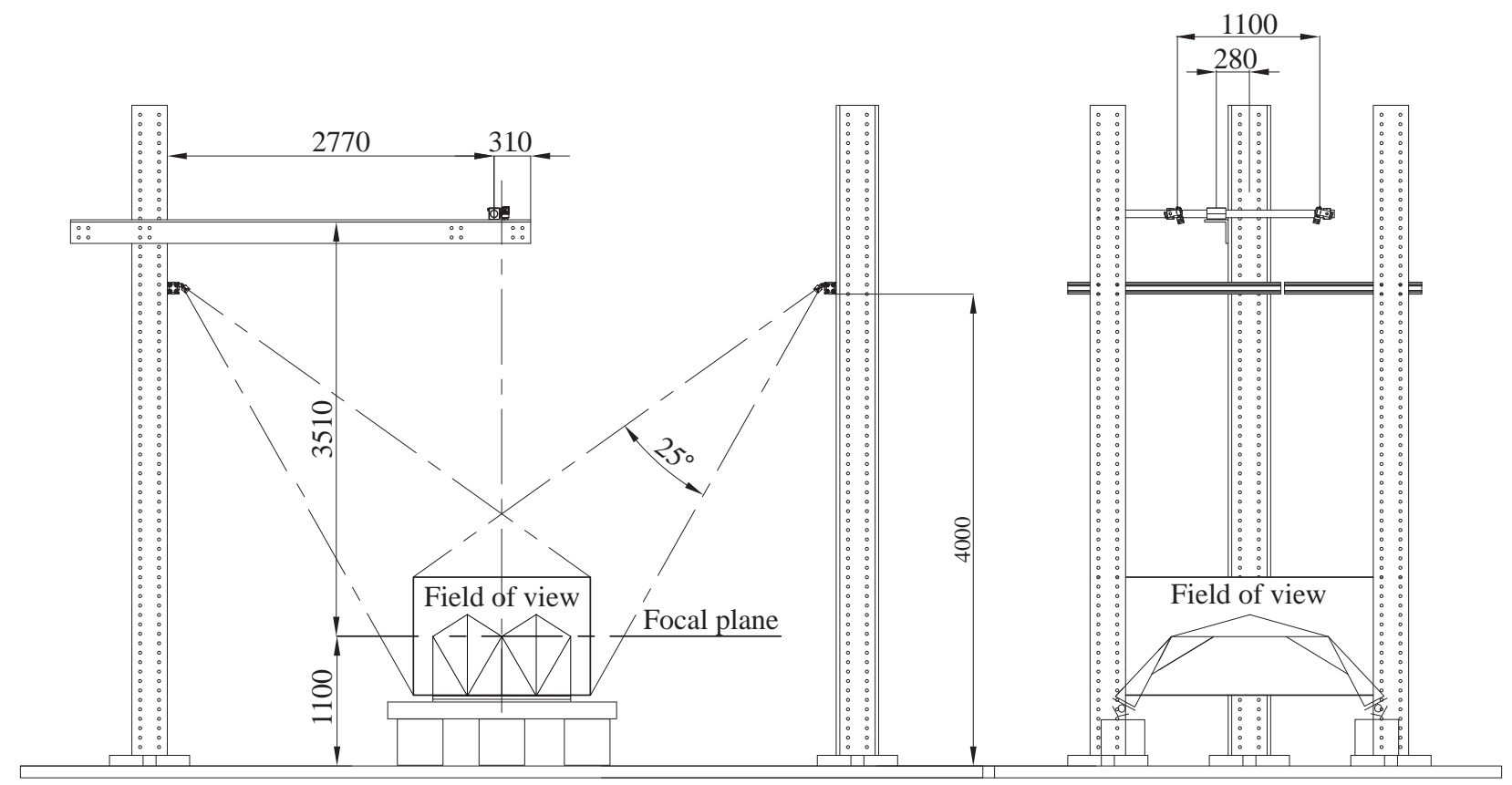

Figure B.19: DIC setup.

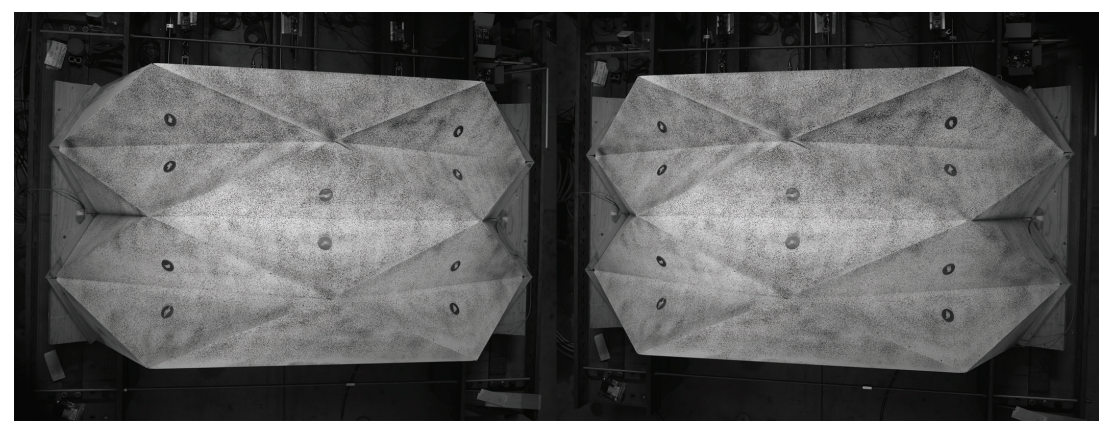

Figure B.20: DIC view form left and right camera.

due to previously explained insufficiently clear view. Values less than $0,005 \mathrm{~mm}$ were targeted for the two plates of the central valley fold, where higher accuracy could be achieved.

A system of pulleys was positioned at each of the three longitudinal lines of loaded elements. It consisted of pulleys attached to the structure at 10 plate geometrical centers, and those attached onto the steel U-beams, which were fixed through the concrete floor slab for keeping the system in position. As shown in (Fig. 9a), two naked edge triangular plates were not loaded during the experiments. This was done in order to avoid high deformations and buckling of long unsupported edges which could lead to unwanted early failure at these regions. Addition- 1015 ally, half triangle plates along the supports were also not 1016 loaded, in favour of reducing the complexity of the setup. ${ }_{1017}$ This decision was supported by the fact that their high in- ${ }_{1018}$ clinations, reduced surface and proximity to the supports 1019 would lead the forces directly into the supports, not having $\quad 1020$ much influence on the global spatial structural behaviour. ${ }_{1021}$ A steel cable, $\phi=5 \mathrm{~mm}$, weaved through each of the three ${ }_{1022}$ systems and was fixed at one end and at the other con- ${ }^{1023}$ nected to a hydraulic linear cylinder, Enerpac RD-910, 1024 through which a displacement-controlled load was intro- ${ }_{1025}$ duced. The preliminary test for evaluating the mechanical ${ }_{1026}$ components of the test rig showed that the available pis- ${ }_{1027}$ 


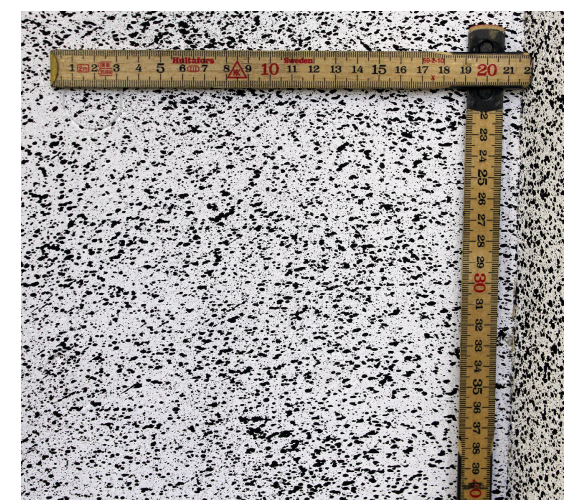

Figure B.21: Speckle pattern with speckle sizes between 1-5mm.

ton stroke of $280 \mathrm{~mm}$ was not sufficient for the planned tests. For that, an extra pulley was added to each of the systems (see Fig.9p) to reduce the amount of generated displacements at the cylinder by half. The loading procedure was established according to [21]. Load was applied in a quasi-static rate using a combination of displacement control and load control methods (Fig. B.22). According to this, seven loading steps were established and implemented automatically by using a PCS 8000 control system by walter+bai ag together with DION7 software package. A detail flowchart of the loading process is presented in (Fig. B.23).

Data acquired from the tests was analysed using both VIC 3D and custom algorithms developed within Matlab. Within the Vic-3D software, the subset size was set to 29 to give an optimal match confidence of 0,01 pixel for a given noise level. The noise level was taken as default of 8 which is suggested to work well for most cameras [28]. 


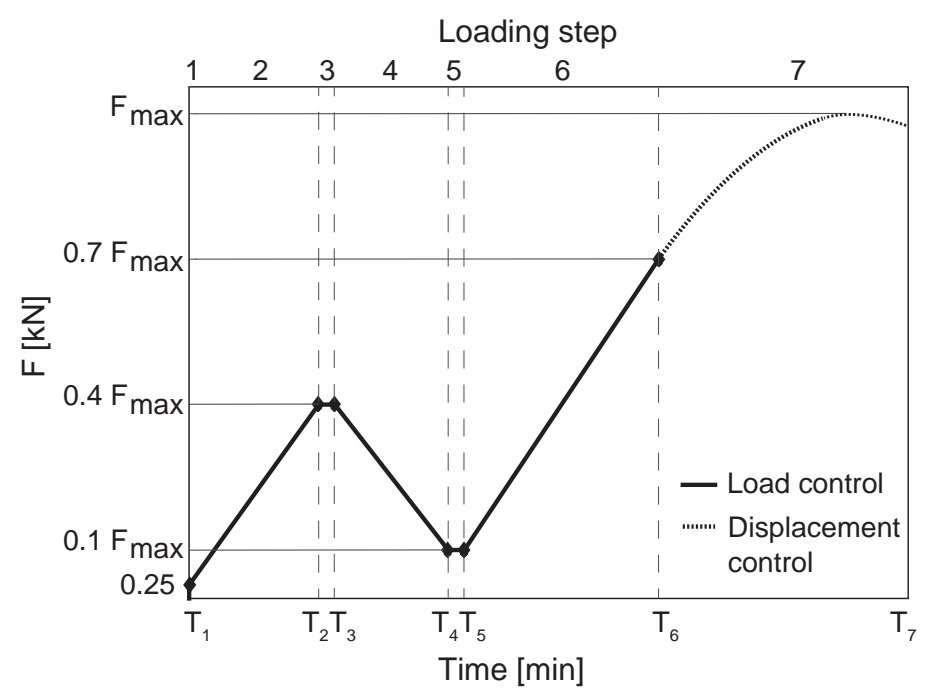

Figure B.22: Loading procedure.

Step 1:

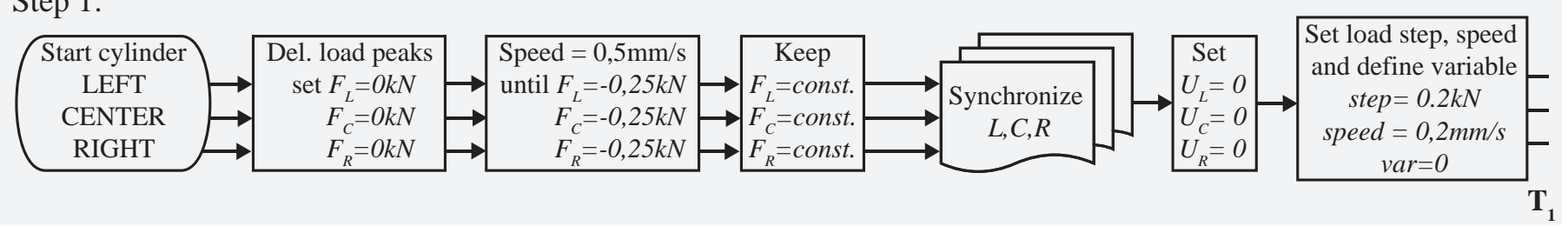

Step 2:

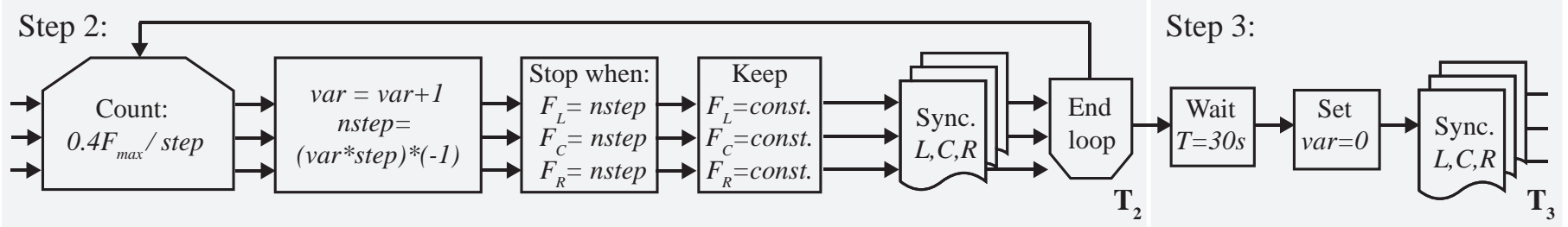

Step 4:
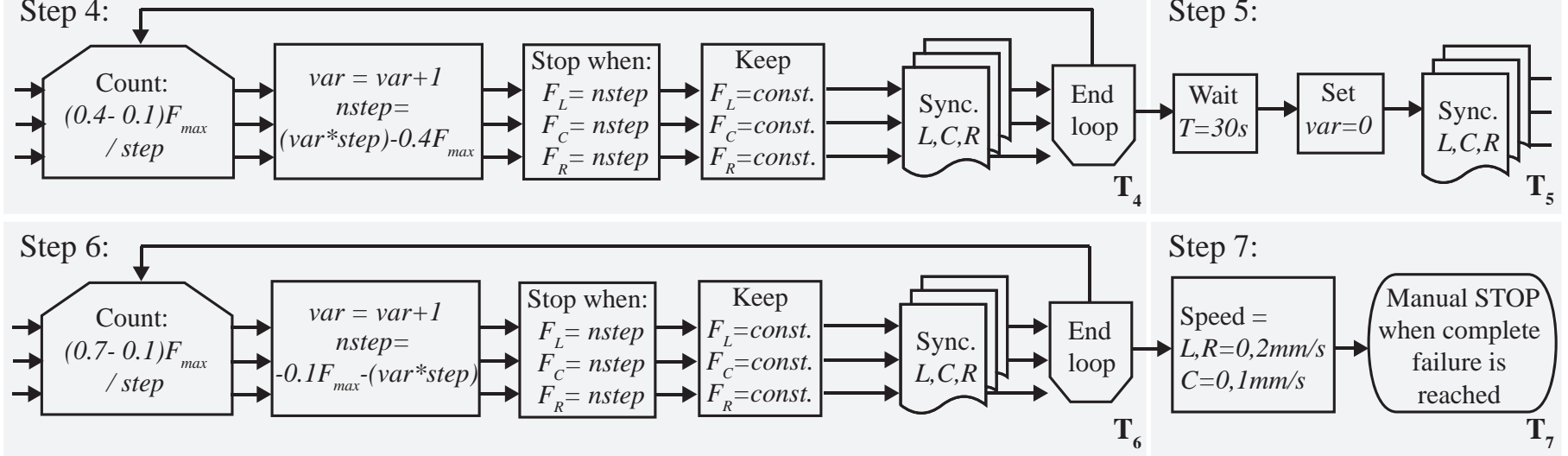

Figure B.23: Test loading process, separated according to the defined seven loading steps. 